\title{
Enhanced Signal Processing Algorithms for Buried Unexploded Ordnance Detection and Location Estimation with Magnetometer and Electromagnetic Induction Measurements
}

\author{
Alan Witten \\ Energy Division \\ Oak Ridge National Laboratory \\ Oak Ridge, Tennessee 37831-6200
}

September 1993

Prepared by the

OAK RIDGE NATIONAL LABORATORY

Oak Ridge, Tennessee 37831

managed by

MARTIN MARIETTA ENERGY SYSTEMS, INC.

for the

U.S. DEPARTMENT OF ENERGY

under Contract No. DE-AC05-84OR21400 


\section{Contents}

List of Figures v

List of Tables $\quad$ vii

Summary $\quad$ ix

1 Introduction $\quad 1$

2 Target Detection and Location Estimation $\quad 3$

2.1 The Maximum Likelihood Estimator . . . . . . . . . . . . . . . . 3

2.2 Data Teriplates for Magnetometry . . . . . . . . . . . . . . 4

2.3 Data Templates for EMI . . . . . . . . . . . . . . . . . 6

2.4 A Normalized Maximum Likelihood Estimator . . . . . . . . . . . . 8

3 Data Simulations and Test Cases $\quad 11$

3.1 EMI Data Template Simulations . . . . . . . . . . . . . . . 11

3.2 EMI Test Case Results . . . . . . . . . . . . . . . . . . . . . . . . . . . . . . .

3.3 Target Dimensionality . . . . . . . . . . . . . 18

4 The Field Study $\quad 21$

5 Results $\quad 31$

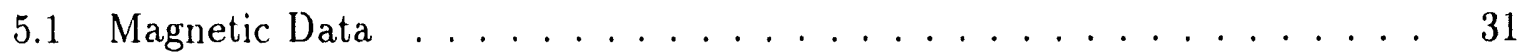

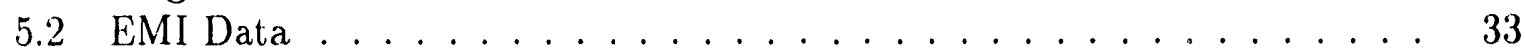

6 Conclusions and Recommendations $\quad 41$ 


\section{List of Figures}

1 A plot of observed and simulated in-phase EMI data as a function of measurement position over the top of a UST. The simulated results are the solid line and the scaled measured values are the ${ }^{*}$ 's. . . . . . . . . .

2 A plot of observed and simulated quadrature EMI data as a function of measurement position over the top of a UST. The simulated results are the solid line and the scaled measured values are the ${ }^{*}$ 's. . . . . . . . . .

3 Contours of constant maximum $\log$ likelihood function as a function of target dimensions for the $800 \mathrm{~Hz}$. radial profile. . . . . . . . . . . . . . 14

4 Plot of the spatial variations in $\log$ likelihood function, displayed as gray levels, for the $800 \mathrm{~Hz}$. UST radial profile. . . . . . . . . . . .

Plot of the spatial variations in log likelihood function, displayed as gray levels, for the scaled $800 \mathrm{~Hz}$. UST radial profile. . . . . . . . . . . . Contours of constant maximum log likelihood function as a function of target dimension for the scaled $800 \mathrm{~Hz}$. radial profile. . . . . . . . . . .

7 Contours of constant maximum $\log$ likelihood function as a function of target dimensions for the $800 \mathrm{~Hz}$. axial profile. . . . . . . . . . .

8 Plot of the spatial variations in $\log$ likelihood function for the axial profile and a $10 \mathrm{ft}$. by $10 \mathrm{ft}$. target. . . . . . . . . . . . . . . 17

$9 \quad$ Plot of the spatial variations in $\log$ likelihood function for the axial profile and an $8 \mathrm{ft}$. by $3 \mathrm{ft}$. target. . . . . . . . . . . . . . .

10 Plot of simulated backscattered data along a line over the top of a spherical

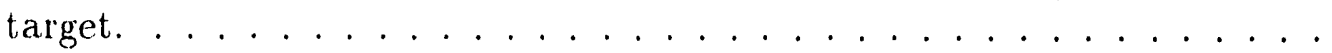

11 Plot of simulated backscattered data along a line over the top of circular target. . . . . . . . . . . . . . . . .

12 Contour plot of perturbations in total magnetic field from a proton precession magnetometer. . . . . . . . . . . . . . . . . 22

13 Contours of in-phase component at $800 \mathrm{~Hz} \ldots \ldots . \ldots 23$

14 Contours of quadrature component at $800 \mathrm{~Hz} \ldots \ldots . \ldots 23$

15 Contours of in-phase component at $1280 \mathrm{~Hz} \ldots \ldots . \ldots 24$

16 Contours of quadrature component at $1280 \mathrm{~Hz} \ldots \ldots . \ldots 24$

17 Contours of in-phase component at $1600 \mathrm{~Hz} \ldots \ldots . \ldots 25$

18 Contours of quadrature component at $1600 \mathrm{~Hz} \ldots \ldots 25$

19 Contours of in-phase component at $3200 \mathrm{~Hz} \ldots \ldots . \ldots 26$

20 Contours of quadrature component at $3200 \mathrm{~Hz} \ldots \ldots . \ldots 26$

21 Contours of in-phase component at $4800 \mathrm{~Hz} \ldots \ldots . \ldots 27$

22 Contours of quadrature component at $4800 \mathrm{~Hz} \ldots \ldots . \ldots 27$

23 Contours of in-phase component at $6400 \mathrm{~Hz} \ldots \ldots . \ldots 28$

24 Contours of quadrature component at $6400 \mathrm{~Hz}$. . . . . . . . . . 28

25 Contours of in-phase component at $9600 \mathrm{~Hz} \ldots \ldots . \ldots 29$

26 Contours of quadrature component at $9600 \mathrm{~Hz}$. . . . . . . . . . . 29 
27 Location of the magnetometer line segments used in signal processing. .

28 Spatial distribution of the log likelihood function, displayed as gray levels, below magnetometer line E2. . . . . . . . . . . . . . . . .

29 Spatial distribution of the log likelihood function, displayed as gray levels, below magnetometer line E3. . . . . . . . . . . . . . . . . . . 34

30 Spatial distribution of the log likelihood function, displayed as gray levels, below magnetometer line $\mathrm{N} 6 . \ldots \ldots \ldots$. . . . . . . . . . . .

31 Spatial distribution of the log likelihood function, displayed as gray levels,

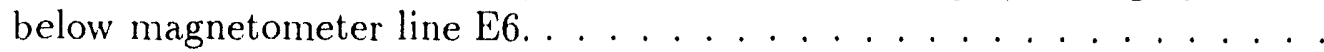

32 Spatial distribution of the log likelihood function, displayed as gray levels,

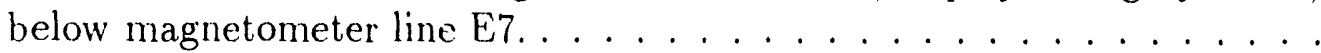

33 Spatial distribution of the log likelihood function, displayed as gray levels, below magnetometer line E8. . . . . . . . . . . . . . . . . 36

34 Spatial distribution of the log likelihood function, displayed as gray levels, below magnetometer line N8. . . . . . . . . . . . . . . . 36

35 Spatial distribution of the log likelihood function, displayed as gray levels, below Target 6 from $1280 \mathrm{~Hz}$. EMI data. . . . . . . . . . . . . . . 38

36 Spatial distribution of the log likelihood function, displayed as gray levels, below Target 6 from $1600 \mathrm{~Hz}$. EMI data. . . . . . . . . . . . . . . .

37 Spatial distribution of the log likelihood function, displayed as gray levels, below Target 7 from $4800 \mathrm{~Hz}$. EMI data. . . . . . . . . . . . . . . 


\section{List of Tables}

1 Locations of magnetic anomalies. . . . . . . . . . . . . . 22

2 Summary of the results of the application of a maximum likelihood estimator to magnetometer data. . . . . . . . . . . . . . 31

3 Summary of the results of the application of a maximum likelihood estimator to EMI data. . . . . . . . . . . . . . . . . . . 37 


\section{Summary}

Enhanced signal processing algorithms have been developed for the detection and location of buried unexploded ordnance using magnetometry and electromagnetic induction (EMI) measurements. These signal processing algorithms are related to those used to image with geophysical diffraction tomography (GD'T) employing wave-based measurements. The underlying relationship of GDT is the Generalized Projection Slice Theorem (GPST) that relates the spatial Fourier transform of acquired data to the spatial Fourier transform of subsurface inhomogeneities of one higher dimension. This relationship can be used to simulate data templates for known targets and, by virtue of the shift property of Fourier transforms, a data simulation need only be computed for one reference target location. All other target locations are generated by an appropriate phase shift. These data templates can be correlated with acquired data to determine the spatial distribution of probable target location. This approach to target detection and location estimation, referred to as a maximum likelihood estimation, can be used to produce an "image" of the likelihood of a specified target's position.

For non wave-based methods, the relationship between data and target characteristics is not strictly associated with Fourier transforms. In the case of magnetometry, the appropriate GPST requires a Fourier-Laplace transform of the target characteristics while the EMI GPS'T is based on an integral transform with a complex wavenumber. Nevertheless, the shift rule for integral transforms can bo invoked to yield GPST's for these tools and the associated computationally efficient maximum likelihood estimators.

The EMI detection algorithm was applied to data acquired at a known underground storage tank site and the algorithms for both magnetometry and EMI were applied to data acquired at the Magnetic Range of the Naval EOD Tech Center in Indian Head, Maryland. In all cases where magnetometer and EMI anomalies, even weak ones, could be measured, the new algorithms estimated the horizontal position and depth with apparent reasonable accuracy. Data templates were constructed for a variety of target sizes in an effort to use the maximum likelihood estimators to estimate the sizes of detected subsurface features. Although there was little information available concerning the size and orientation of most targets, it is believed that size estimation is not as accurate as position estimation. The likely cause of this is that simplified GPST's based on twodimensional targets were used rather than the realistic representation of these targets as three-dimensional. 


\section{Introduction}

It is only within about the past decade that there has been a significant effort in the development, and application of geophysical methods to shallow subsurface investigations. Most of this effort has been directed towards problems associated with environmental remediation but other problems, such as unexploded ordnance and tunnel detection, have also become important applications for geophysics. The tools of choice in shallow site investigations are ground penetrating radar (GPR), magnetometry, and electromagnetic induction (EMI) because they can rapidly survey large spatial extents with the high measurement density required at the subject sites. Seismic methods are employed to a lesser extent because they are much slower to deploy. There is a great body of literature associated with the processing of seismic signals resulting from the fact that seismics has been the mainstay of the petroleum exploration industry for almost a century. Because of their more recent introduction, very little consideration has been given, until quite recently, to the development of signal processing algorithms for GPR, magnetometry or EMI. For the most part, visual inspection of data acquired with these tools is used to identify the presence of leatures of interest.

Recently, techniques have been developed and applied to shallow geophysical measurements that provide results that are more quantitative and less reliant on interpretive insight. The earliest of these methods is geophysical tomography (GI) that, in its original embodiment, assumes straight ray paths and backprojects acquired data along these straight rays to form a tomographic image of a two-dimensional cross-section. These methods use signal processing procedures almost identical to those used in CT scanners employed in diagnostic medicine. More recently, iterative GT methods were developed to more properly account for the ray bending known to occur in geologic material. Most recently, G'T has been generalized to more rigorously account for the redistribution of amplitude and phase (diffraction) that occurs at the longer wavelengths required in geophysical investigations. 'This technique, known as geophysical diffraction lomography (GI)'T), is based on an analytic relationship between the one (two)-dimensional spatial Fourier transform of the measured data and the two (three)-dimensional spatial Fourier transform of subsurface inhomogeneities. This relationship, referred to as the Generalized Projection Slice Theorem (GPST) is analogous to the equation of a holographic lens. (iD)T has been successfully applied to radar and scalar seismic (acoustic) data to yield high-resolution images of buried features. It has been recognized that the GPST represents a relationship between target characteristics and location that can be used in a computationally efficient manner to address the problem of estimating the presence and location of a target having known characteristics. In a manner similar to the application of Ci'T or GDT, this detection procedure produces an image of the spatial variations in a statistical quantity, rather than a physical quantity, known as the log likelihood function. Increasing values of the log likelihood function are associated with a better match to target location and characteristics. When this value exceeds a specified threshold, the target can be assumed to be present with its location coinciding with the location of the 
peak value of this function. This method is called geophysical diffraction tomography maximum likelihood estimation (GDTMLE) and it has been employed in target detection using both radar and acoustic measurements.

In this study, GDTMLE signal processing algorithms are developed and tested for EMI and magnetometry measurements for potential use at buried unexploded ordnance sites. In magnetometry, measurements are made of perturbations in the Earth's magnetic potential that result from buried ferromagnetic inclusions. EMI detects the presence of subsurface conductors through the use of two coils. The first, or primary coil, creates a magnetic field in the subsurface that, in turn, induces currents in any subsurface conductors. These induced currents create a secondary field that is detected by a second coil associated with the EMI tool. There are many types of EMI tools, those most commonly used and considered in this study employ two coils at the opposite ends of a short boom. These tools are light weight and are carried by one individual across a site.

The development of GDTMLE algorithms for magnetometer and EMI measurements are a significant departure from previous efforts in this area. To date, GDTMLE algorithms have only been developed and used for wave-based measurements while, in this study, they are considered for the magnetostatic and eddy current problems associated with magnetometry and EMI, respectively. These new algorithms were field tested at the Naval EOD Tech Center in Indian Head, Maryland. Data were acquired with two magnetometers and two EMI tools. One magnetometer was a proton precession magnetometer and the other was a gradient flux gate magnetometer. Both magnetometers are commercially available; however, the ordnance detector flux gate magnetometer was modified by Geophex, Ltd. of Raleigh, North Carolina to incorporate an analog-to-digital converter and a digital display. One EMI tool is the commercially available Geonics EM 31 and the second is the one of a kind GEM 1 developed by Geophex. The planning and execution of the Indian Head field study was done in collaboration with staff from Geophex and with support from Mr. Jim Ursic of the U.S. EPA, Region 5.

The next section provides the derivation of the maximum likelihood estimator and the GPST's specific to both magnetometry and EMI. Section 3 presents simulations and test cases directed towards the assessment and performance of the developed signal processing algorithms. The Indian Head field study is described and acquired data presented in Sect. 4. The results of the application of the new signal processing algorithms is given in Sect. 5 and Sect. 6 presents conclusions and recommendations for further studies. 


\section{Target Detection and Location Estimation}

The problem of detecting a known target from surface geophysical measurements can be approached in a number of ways. The simplest and most commonly used procedure is inspection of the unprocessed (acquired) data for anomalies that can be recognized as subsurface inhomogeneities. Using ground penetrating radar, for example, the presence of a hyperbola in the plot of amplitude versus time for adjacent surface antenna positions is evidence of a buried isolated inclusion. In magnetometry and electromagnetic induction (EMI), spatially correlated variation in measurements are similarly suggestive of isolated subsurface inclusions. One of the main problems with buried target location and identification when using magnetometer or EMI data is the ambiguity among size, contrast and depth of buried targets. For instance, an inhomogeneity that produces a broad "footprint," on the ground surface could be interpreted to be either a strong, small and decp target or a weak, large and shallow target.

This study develops and tests more quantitative procedures for buried target detection and identification. The basic concept is that of maximum likelihood estimation (MLE) which is essentially a template matching method coupled with an efficient way of computing these data templates using an analytic relationship between measured data and the location and characteristics of specified inhomogeneities known as the generalized projection slice theorem (GPST). The following subsections present the MLE concept and the specific GPST's for a magnetometry and a EMI measurement configuration.

It is important to note here that, for simplicity, all derivations are based on twodimensional targets lying in a vertical cross-section with one-dimensional measurements taken over a line along the ground surface. The generalization to three dimensions is straightforward for magnetometry but more difficult for EMI. The implications of treating three-dimensional inhomogeneities as two dimensional targets are discussed in Sect. 3.

\subsection{The Maximum Likelihood Estimator}

Defining the data measured along a line, $\ell$, on the ground surface as $u(\ell)$ and the data that would be measured if a known target were located at position $\mathbf{x}_{0}=\left(x_{0}, z_{0}\right)$ in a vertical cross-section as $v\left(\ell ; \mathbf{x}_{0}\right)$, an error term can be defined in the least squares sense as

$$
\mathcal{E}\left(\mathbf{x}_{0}\right)=\int d \ell\left|u(\ell)-v\left(\ell ; \mathbf{x}_{0}\right)\right|^{2}
$$

In practice, the error $\mathcal{E}$ will never be zero as a result of noisy data, imprecise target characterization, or a poorly matched target position $\mathbf{x}_{0}$. The best estimate of target location will be the position $\mathbf{x}_{0}$ that minimizes $\mathcal{E}$. Expanding the square indicated in Eq. (1)

$$
\mathcal{E}\left(\mathbf{x}_{0}\right)=\Re \int d \ell\left[u u^{*}-2 u v^{*}+v v^{*}\right]
$$


where the superscript asterisk denotes the complex conjugate and $\Re$ means the real part. It is recognized that the first term, $u u^{*}$, is independent of target location, $\mathbf{x}_{0}$, and consequently does not impact the minimization of $\mathcal{E}$. For this reason, this term can be dropped from Eq. (2), and the resulting equation multiplied by -1 to yield the $\log$ likelihood function

$$
F_{L}\left(\mathbf{x}_{0}\right)=\Re \int d \ell\left[2 u v^{*}-v v^{*}\right],
$$

where the best estimate of target location is now (by virtue of the sign change) the $\mathbf{x}_{0}$ that maximizes $F_{L}$. For reasons that will become clear later, by defining the spatial Fourier transform of a function $f$ as

$$
\hat{f}(\kappa)=\int d \ell e^{-i \kappa \ell} f(\ell)
$$

Parseval's theorem can be used to rewrite Eq. (3) in the form

$$
F_{L}\left(\mathbf{x}_{0}\right)=\Re \int d \kappa\left[2 \hat{u}(\kappa) \hat{v}^{*}\left(\kappa, \mathbf{x}_{0}\right)-\hat{v}\left(\kappa, \mathbf{x}_{0}\right) \hat{v}^{*}\left(\kappa, \mathbf{x}_{0}\right)\right] .
$$

Equations (1), (2), (3), and (5) are functionally equivalent for estimating the existence and location of a specified target. This target can be deemed to be present if the value of $F_{L}(\mathcal{E})$ exceeds (is below) a certain threshold and, if present, its location is estimated to be the $\mathrm{x}_{0}$ that maximizes (minimizes) $F_{L}(\mathcal{E})$. The drawback to using any of these equations is the extreme computational and data storage overhead associated with the need for data templates, $u\left(\ell ; \mathbf{x}_{0}\right)$, at all possible target locations $\mathbf{x}_{0}$. Furthermore, should there be any uncertainty in the size, shape and/or orientation of the target; multiple data templates would be required for every location $\mathbf{x}_{0}$. The following subsections present an approach to the generation of data templates that requires the computation of these templates at only a single reference target position and, as a consequence, minimizes computational and storage requirements.

\subsection{Data Templates for Magnetometry}

The computationally efficient procedure for generating data templates requires the derivation of an appropriate GPST which is an analytic relationship between the data that would be measured for a target centered at some position $\mathbf{x}_{0}$ and the target characteristics. This derivation can be accomplished by first considering the equation governing magnetometry measurements. A magnetometer measures the magnitude of the Earth's magnetic potential $\bar{\phi}$ at some point in space. This potential is governed by

$$
\nabla \cdot[\bar{\mu} \nabla \bar{\phi}]=0
$$

where $\bar{\mu}$ is the magnetic permeability at some point in space $\mathbf{x}$. By defining

$$
\ddot{\mu}=\mu_{0}+\epsilon \mu(\mathbf{x})
$$


and

$$
\bar{\phi}=\phi_{0}(\mathbf{x})+\epsilon \phi(\mathbf{x})
$$

where the background magnetic permeability $\mu_{0}$ is taken to be constant, the perturbed potential $\phi$ is associated with buried magnetic inclusions $\mu(\mathbf{x})$, and $\epsilon$ is a small parameter included explicitly to establish that buried inhomogeneities produced only a small change in measured magnetic potential; Eq. (6) can be linearized to give

$$
\nabla^{2} \phi=-\nabla \cdot\left(O(\mathbf{x}) \nabla \phi_{0}\right)
$$

In Eq. (9), $O(\mathbf{x})=\mu(\mathbf{x}) / \mu_{0}$ is referred to as the object function and $\phi_{0}$ is the Earth's ambient magnetic potential associated with a constant permeability $\mu_{0}$. The term $\nabla \phi_{0}$ in this equation is the Earth's vector magnetic field at some point $\mathbf{x}$ which can be represented by $\mathbf{B}_{0}(\mathbf{x})$.

Equation (9) can be expressed in the form of the integral equation

$$
\phi(\mathbf{r})=\frac{\mathbf{B}_{0}}{4 \pi} \cdot \int d \boldsymbol{\xi} G(\mathbf{r}-\boldsymbol{\xi}) \nabla_{\boldsymbol{\xi}} O(\boldsymbol{\xi}),
$$

where $G$ is the Green's function for Laplace's equation. Equation (10) is the first step in the development of a data template since it relates $\phi$ at a location $\mathbf{r}$, that can be taken to be a measurement point, to the target location and characteristics defined by $O$. At this point, however, computations of $\phi$ must be made at every possible target location. It remains to render this relationship in a more computationally efficient form. To achieve this end, the Green's function can be expressed in terms of the integral representation

$$
G(\mathbf{r}-\boldsymbol{\xi})=\int \frac{d \alpha}{|\alpha|} e^{(-|\alpha| \hat{\mathbf{z}}+i \alpha \hat{\mathbf{x}}) \cdot(\mathbf{r}-\boldsymbol{\xi})}
$$

where $\hat{\mathbf{x}}$ and $\hat{\mathbf{z}}$ are unit vectors in the horizontal and vertically upward directions, respectively. Taking the measurement position to be $\mathbf{r}=(\ell, 0)$, substituting Eq. (11) into Eq. (10), and Fourier transforming the resulting equation with respect to the position $\ell$ along the measurement line $[\mathrm{Eq}$. (4)] gives

$$
\hat{\phi}(\kappa)=\frac{\mathbf{B}_{0}}{2|\kappa|} \cdot \int d \boldsymbol{\xi} \nabla_{\boldsymbol{\xi}} O(\boldsymbol{\xi}) e^{-i \mathbf{K} \cdot \boldsymbol{\xi}}=\frac{i \mathbf{B}_{0} \cdot \mathbf{K}}{2|\kappa|} \int d \boldsymbol{\xi} O(\boldsymbol{\xi}) e^{-i \mathbf{K} \cdot \boldsymbol{\xi}},
$$

where

$$
\mathbf{K}=\kappa \hat{\mathbf{x}}+i|\kappa| \hat{\mathbf{z}}
$$

The final form of the data template is obtained by recognizing that Eq. (12) relates the one-dimensional spatial Fourier transform of the measured perturbed potential to the two-dimensional spatial Fourier-Laplace transform of the object function $O$. Taking $O_{0}(\mathbf{x})$ to be the object function of a target centered at a reference location (here taken to be the origin), the Fourier-Laplace transform $\hat{O}\left(\mathbf{K} ; \mathbf{x}_{0}\right)$ of the object function of a target centered at $\mathbf{x}_{0}$

$$
\hat{O}\left(\mathbf{K}, \mathbf{x}_{0}\right)=\int d \boldsymbol{\xi} O\left(\boldsymbol{\xi}, \mathbf{x}_{0}\right) e^{-i \mathbf{K} \cdot \boldsymbol{\xi}}
$$


is related to $\hat{O}_{0}(\mathbf{K})$ by the shift rule as

$$
\hat{O}\left(\mathbf{K}, \mathbf{x}_{0}\right)=\hat{O}_{0}(\mathbf{K}) e^{-i \mathbf{K} \cdot \mathbf{x}_{0}}
$$

By defining the measurements $u(\ell)[\mathrm{Eq}$. (1)] to be the perturbation relative to background of the magnetic potential, the appropriate data template for use in $\mathrm{Eq}$. (5) is obtained by substitution of Eq. (15) into Eq. (12) to give

$$
\hat{v}\left(\kappa ; \mathbf{x}_{0}\right)=\frac{i \mathbf{B}_{0} \cdot \mathbf{K}}{2|\kappa|} \hat{O}_{0}(\mathbf{K}) e^{-i \mathbf{K} \cdot \mathbf{x}_{0}},
$$

where $\mathbf{K}$ is defined in $\mathrm{Eq}$. (13).

Equation (16) is the desired computationally efficient data template in that this template is only computed once at a specified reference location with the data templates obtained for all other locations incorporated by the phase shift $-i \mathbf{K} \cdot \mathbf{x}_{0}$. Equations (12) and (16) are the GPST's for magnetometry measurements relating the one-dimensional spatial Fourier transform of the data (or data template) to the two-dimensional spatial Fourier-Laplace transform of the object function (target characteristics). To exploit this relationship, the form of the log likelihood function given by Eq. (5) is used.

\subsection{Data Templates for EMI}

The derivation of data templates for EMI follows a similar procedure to that employed for magnetometry. A GPST is developed for a particular measurement geometry based on the governing equation for EMI measurements

$$
\nabla^{2} \hat{\phi}-\sigma(\mathbf{x}) \mu_{0} \frac{\partial \hat{\phi}}{\partial t}=f(\mathbf{x}, t)
$$

where the magnetic permeability can be taken to be the constant background value $\mu_{0}$, $\sigma(\mathbf{x})$ is the spatially variable electrical conductivity, $f$ is the source term characterizing the spatial and temporal variations of the primary coil, and $t$ is time. By defining the object function to be

$$
O(\mathbf{x})=\epsilon\left(\sigma(\mathbf{x})-\sigma_{0}\right) / \sigma_{0}
$$

where $\sigma_{0}$ is a constant background conductivity, and

$$
\hat{\phi}(\mathbf{x})=\phi_{0}(\mathbf{x})+\epsilon \phi(\mathbf{x})
$$

Eq. (17) can be linearized to yield the two governing equations

$$
\nabla^{2} \phi_{0}-\sigma_{0} \mu_{0} \frac{\partial \phi_{0}}{\partial t}=f(\mathbf{x}, t)
$$

and

$$
\nabla^{2} \phi-\sigma_{0} \mu_{0} \frac{\partial \phi}{\partial t}=\sigma_{0} \mu_{0} O(\mathbf{x}) \frac{\partial \phi_{0}}{\partial t}
$$


Equation (20) models the potential produced by the primary coil and Eq. (21) similarly models the perturbed potential resulting from conducting or insulating inhomogeneities defined by $O$.

The most commonly used EMI tools employ two coils at opposite ends of a short boom. These devices operate in the frequency domain so that the measured quantity is

$$
\tilde{\phi}(\mathbf{x}, \omega)=\int_{0}^{\infty} d t \phi(\mathbf{x}, t) e^{i \omega !}
$$

This quantity is a single complex number with the real and imaginary parts of $\tilde{\phi}$ referred to as quadrature and in-phase, respectively. Applying the temporal Fourier transform operator $[\mathrm{Eq}$. (22)] to Eq. (21), the integral equation governing $\tilde{\phi}$ at some measurement position $\mathbf{r}$ can be written

$$
\tilde{\phi}(\mathbf{r})=-\frac{i \omega \sigma_{0} \mu_{0}}{4 \pi} \int d \boldsymbol{\xi} G(\mathbf{r}-\boldsymbol{\xi}) O(\boldsymbol{\xi}) \tilde{\phi}_{0}(\boldsymbol{\xi},
$$

where $G$ is the Green's function for the two-dimensional Helmholtz equation given by

$$
G(\mathbf{r}-\boldsymbol{\xi})=i \pi H_{0}^{(1)}(k|\mathbf{r}-\boldsymbol{\xi}|)=i \int \frac{d \alpha}{\sqrt{k^{2}-\alpha^{2}}} e^{i\left(\sqrt{k^{2}-\alpha^{2}} \hat{\mathbf{z}}+\alpha \hat{\mathbf{x}}\right) \cdot(\mathbf{r}-\boldsymbol{\xi})}
$$

$H_{0}^{(1)}$ is the zeroth order Hankel function of the first kind, and $k=\sqrt{-i \sigma_{0} \mu_{0} \omega}$.

Taking $\tilde{\phi}_{0}$ to be the potential generated from a monopole source collocated with the receiving coil, $\tilde{f}(\mathbf{x}, \omega)=-4 \pi \delta(\mathbf{x}-\mathbf{r})$, and $\tilde{\phi}_{0}(\boldsymbol{\xi})=G(\boldsymbol{\xi}-\mathbf{r})$. Substituting this form of $\tilde{\phi}_{0}$ into Eq. (23) gives

$$
\tilde{\pi}(\mathbf{r})=\frac{k^{2}}{4 \pi} \int d \boldsymbol{\xi} G^{2}(\mathbf{r}-\boldsymbol{\xi}) O(\boldsymbol{\xi}) .
$$

In order to follow the procedure developed in the preceding subsection, it is necessary to express the square of the Green's function $G^{2}$, rather than the Green's function itself, in a Weyl expansion [Eq. (24)]. No exact closed form expression for such an expansion has been found, however; a full asymptotic representation can be written as

$$
G^{2}(\mathbf{r}-\boldsymbol{\xi})=\frac{i}{8 \pi^{2} k} e^{i \pi / 4} \int \frac{d \alpha}{\sqrt[4]{4 k^{2}-\alpha^{2}}} e^{i\left(\sqrt{4 k^{2}-\alpha^{2}} \hat{\mathbf{z}}+\alpha \hat{\mathbf{x}}\right) \cdot(\mathbf{r}-\boldsymbol{\xi})} \sum_{n=0}^{\infty} \frac{A_{n}(k, \alpha)}{|\eta|^{n+\frac{1}{2}}}
$$

where

$$
A_{n}(k, \alpha)=\frac{i^{n} \Gamma\left(n+\frac{1}{2}\right)}{\sqrt{2 \pi}} \sum_{m=0}^{n} \frac{(-1)^{m} \Gamma\left(m+\frac{1}{2}\right) P_{n-m}\left(-\frac{\sqrt{4 k^{2}-\alpha^{2}}}{2 k}\right)}{2^{m} \Gamma(m+1)\left[\sqrt{4 k^{2}-\alpha^{2}}\right]^{m}(2 k)^{n-m}},
$$

$\Gamma$ is the Gamma function, $P_{n-m}$ is the Legendre Polynomial of order $n-m$, and $\eta=\boldsymbol{\xi} \cdot \hat{\mathbf{z}}$. It is important to note, at this point, that the series defined by Eqs. (26) and (27) converge rapidly for $|k|$ large; however, this quantity is quite small for EMI and this series may not converge at all. Ignoring this fact, the final form of the GPST for EMI is obtained 
by substituting Eq. (26) into Eq. (25), taking $\mathbf{r}=(\ell, 0)$, and Fourier transforming the resulting equation with respect to $\ell$

$$
\hat{\phi}(\kappa)=\int d \ell e^{-i \kappa \ell} \dot{\phi}(\mathbf{r})
$$

to yield

$$
\hat{\phi}(\kappa)=\frac{i k}{16 \pi^{2} \sqrt[4]{4 k^{2}-\kappa^{2}}} e^{i \pi / 4} \sum_{n=0}^{\infty} A_{n}(k, \kappa) \mathcal{O}^{n}(\mathbf{K}),
$$

where

$$
\begin{gathered}
\mathcal{O}^{n}(\boldsymbol{\xi})=\frac{O(\boldsymbol{\xi})}{|\eta|^{n+\frac{1}{2}}} \\
\hat{\mathcal{O}}^{n}(\mathbf{K})=\int d \boldsymbol{\xi} \mathcal{O}^{n}(\boldsymbol{\xi}) e^{-i \mathbf{K} \cdot \boldsymbol{\xi}}
\end{gathered}
$$

and

$$
\mathbf{K}=\kappa \hat{\mathbf{x}}+\sqrt{4 k^{2}-\kappa^{2}} \hat{\mathbf{z}} .
$$

Equation (29) is the desired GPST relating the one-dimensional Fourier transform of the measured perturbed potential along a line $\ell$ to the two-dimensional spatial Fourier transform of the object function divided by various powers of depth $|\eta|$. The data templates for EMI can be generated by using the shift rule to relate $\hat{\mathcal{O}}^{n+\frac{1}{2}}\left(\mathbf{K}, \mathbf{x}_{\mathbf{0}}\right)$ to this same quantity at some reference location, taken here to be the origin $\hat{\mathcal{O}}_{0}^{n+\frac{1}{2}}(\mathbf{K})=\hat{\mathcal{O}}^{n+\frac{1}{2}}(\mathbf{K}, 0)$. This gives

$$
\hat{v}\left(\kappa ; \mathbf{x}_{0}\right)=\frac{i k}{16 \pi^{2} \sqrt[4]{4 k^{2}-\kappa^{2}}} e^{i \pi / 4}\left[\sum_{n=0}^{\infty} A_{n} \hat{\mathcal{O}}^{n}(\mathbf{K})\right] e^{-i \mathbf{K} \cdot \mathbf{x}_{0}},
$$

where

$$
\hat{\mathcal{O}}^{n}(\mathbf{K})=\int d \boldsymbol{\xi} \frac{O_{0}(\boldsymbol{\xi})}{|\eta|^{n+\frac{1}{2}}} e^{-i \mathbf{K} \cdot \boldsymbol{\xi}}
$$

Equation (33) is the data template used in Eq. (5) for the log likelihood function. This data template need only be computed at a single location with its value at all other locations explicitly represented by the phase shift $-i \mathbf{K} \cdot \mathbf{x}_{0}$.

\subsection{A Normalized Maximum Likelihood Estimator}

The log likelihood function given by Eq. (5) can be used with the data templates given by Eqs. (16) and (33) for magnetometer and EMI data, respectively. The use of these data templates may pose problems, in practice, since a contrast must be specified for the object function. For magnetometry, this contrast is the ratio of the targets magnetic permeability to the background permeability and, for EMI, the contrast is the ratio of the target's conductivity to the background conductivity minus one. In most situations involving the detection and location of unexploded ordnance, targets will have a complicated, spatially variable contrast that will be difficult to specify. To deal with such 
situations a normalized log likelihood function can be employed that is based on a unit contrast.

To derive this normalized log likelihood function it is first recognized that, with the approximation used to linearize the governing equations, the data templates are a linear function of contrast. Thus, for a data template $v$ and contrast $\beta$,

$$
v=\beta v^{\prime}
$$

where $v^{\prime}$ is the data template using a contrast of unity. Substituting this relationship into Eq. (5) gives

$$
F_{L}\left(\mathbf{x}_{0}\right)=\Re \int d \kappa\left[2 \beta \hat{u} \hat{v}^{\prime *}-\beta^{2} \hat{v}^{\prime} \hat{v}^{\prime *}\right] .
$$

For a fixed $\mathbf{x}_{0}, F_{L}$ will be maximized for

$$
\frac{\partial F_{L}}{\partial \beta}=\Re \int d \kappa\left[2 \hat{u} \hat{v}^{\prime *}-2 \beta \hat{v}^{\prime} \hat{v}^{\prime *}\right]=0
$$

or

$$
\beta=\frac{\Re \int \hat{u} \hat{v}^{\prime *} d \kappa}{\int \hat{v}^{\prime} \hat{v}^{\prime *} d \kappa} .
$$

Substituting Eq. (38) into Eq. (36) the normalized log likelihood function becomes

$$
F_{L}\left(\mathbf{x}_{0}\right)=\frac{\left(\Re \int \hat{u} \hat{v}^{\prime *} d \kappa\right)^{2}}{\int \hat{v}^{\prime} \hat{v}^{\prime *} d \kappa}
$$

An alternative log likelihood function can be defined as the square root of Eq. (39)

$$
F_{L}\left(\mathbf{x}_{0}\right)=\frac{\Re \int \hat{u} \hat{v}^{\prime *} d \kappa}{\sqrt{\int \hat{v}^{\prime} \hat{v}^{\prime *} d \kappa}} .
$$

While the use of Eqs. (39) or (40) provide a means to avoid the need to specify a target contrast and instead use a unit target contrast, the computed optimized contrast given by Eq. (38) is spatially variable. This approach will given a $F_{L}$ that will be a maximum at the proper target location; however, it will also adjust the value of $\beta$ at all other positions $\mathbf{x}_{0}$ that will locally maximize $F_{L}$. Thus the result of employing either Eq. (39) or (40) will be to "blur" the spatial variations in the log likelihood function. 


\section{Data Simulations and Test Cases}

In the previous section, data templates were developed for use in the log likelihood formulation for target detection. In deriving these data templates, a number of simplifying assumptions were made in order to obtain the necessary analytic relationships given by the GPST's. This section presents some data simulations directed towards the understanding of the validity of these assumptions, specifically the representation of a three-dimensional target as its projection onto a vertical plane and the asymptotic series representation for EMI [Eq. (33)], and to assess the accuracy/uncertainty in target size discrimination and location estimation within the maximum likelihood procedure.

\subsection{EMI Data Template Simulations}

One major uncertainty in the data template formulation developed in Sect. 2.3 for EMI is the validity of the asymptotic series defined by Eqs. (26) and (27). It is known that this series converges rapidly for ground penetrating radar (GPR) applications. In fa ', for frequencies typical of GPR only the first term $(n=0)$ need be used. For EMI applications, frequencies range from hundreds of Hertz to about 10 kilohertz as compared to the megahertz range commonly employed in GPR. At the low frequencies of EMI, the series representation for the square of the Green's function will converge very slowly, if at all.

In order to assess the validity of this representation, data is modeled using Eq. (33) retaining a small number of terms in this series and the result is compared to observed data. Data used in this comparison were acquired over a test site in North Carolina containing a buried underground storage tank (UST). The top of the UST is about 3 $\mathrm{ft}$. below the ground surface. The tank is approximately cylindrical having an estimated length of $8 \mathrm{ft}$. and diameter of $3 \mathrm{ft}$. with the long axis parallel to the ground surface. Data were acquired using the Geophex GEM 1 EMI tool. The $30 \mathrm{ft}$. by $30 \mathrm{ft}$. site was grided into $5 \mathrm{ft}$. square measurement cells and EMI measurements (perturbed potential) wes $e$ acquired at the center of each cell at frequencies of 800,3200 , and $9600 \mathrm{~Hz}$. A comparison between simulated and measured data is made along a line over the top of the UST in a direction perpendicular to the axis of the UST at $800 \mathrm{~Hz}$. In two dimensions, the target cross-section below this line is taken to be a circle with a $3 \mathrm{ft}$. diameter. For the simulation model, only terms in the series [Eqs. (27), (33), and (34)] of order one over the depth to the $2+1 / 2$ power or lower are retained. One significant difference between measurements and simulations is that, for the simulations, the difference between in-phase and quadrature components are everywhere quite small (less than 1\%) while, for the measurements the peak in-phase value is about a factor of seven larger than the peak quadrature value. This difference between observed in-phase and quadrature suggests that the object function used in the simulations should properly be modeled as a complex rather than real quantity. To avoid trying to empirically determine the best data matching ratio of real and imaginary parts of $O$, the in-phase measurements were 
scaled to the quadrature measurements and both the measured and simulated results were normalized consistent with the contrast normalization procedure given in Sect. 2.4. In addition, the measured values were shifted slightly (less than $1 \mathrm{ppm}$ ) so as to better overlay comparative plots of simulated versus measured data. These comparisons are shown in Figs. (1) and (2) for in-phase and quadrature, respectively. It is seen that the data template models the actual data quite well with the major differences occurring

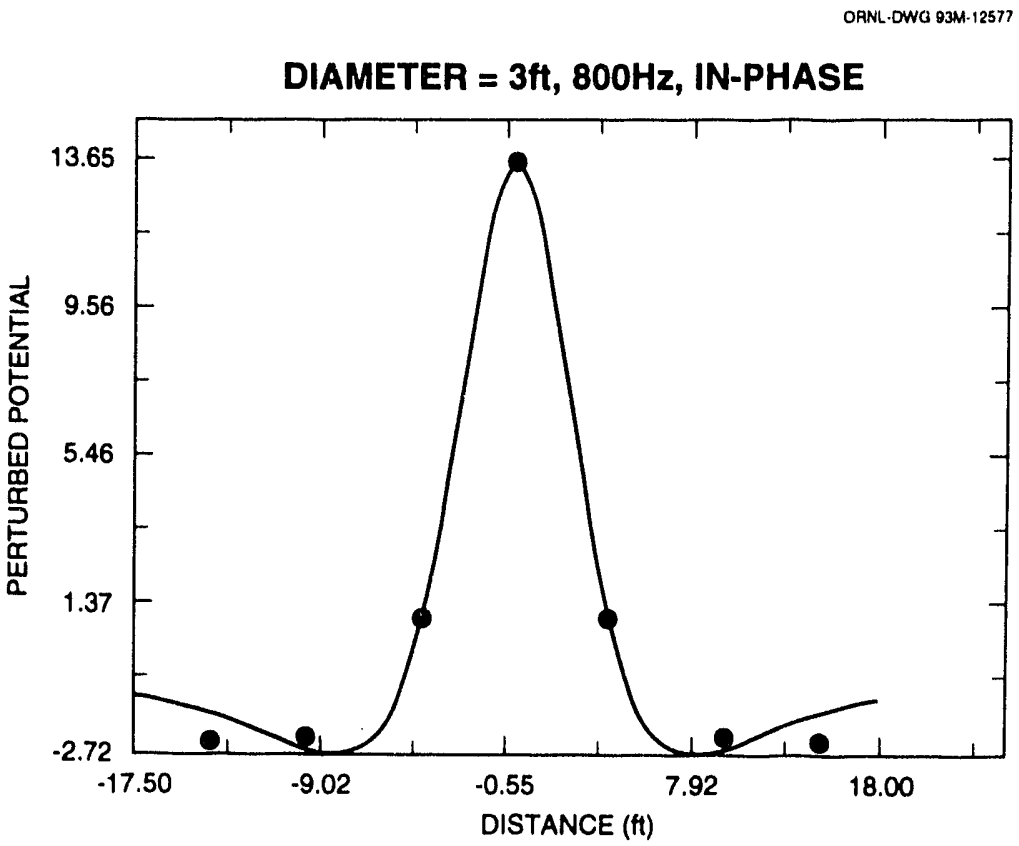

Figure 1: A plot of observed and simulated in-phase EMI data as a function of measurement position over the top of a UST. The simulated results are the solid line and the scalcd measured values are the ${ }^{*}$ 's.

away from the peaks. Comparisons have not been made for other frequencies; however, the truncated series approximation used in the data template model should improve with increased frequency. Thus, the comparison made at $800 \mathrm{~Hz}$ is considered to be a worst case.

\subsection{EMI Test Case Results}

The data acquired over the UST described in the previous subsection were used in the maximum likelihood estimator given by Eq. (40) based on the EMI data template model [Eq. (33)]. The target specification used in these test cases was a rectangle to allow for the fact that the target may not be circular in cross-section. An additional motivation for using rectangular rather than circular cross-sections is that the spatial Fourier transforms of sucis shapes can be expressed analytically as opposed to numerical computations required for circular or more complex shapes. 
A bank of 25 data templates were computed for rectangular targets having horizontal and vertical extents ranging from 2 to $10 \mathrm{ft}$. Each data template represents a particular rectangular target size. For each template, the maximum likelihood estimator was applied to the $800 \mathrm{~Hz}$. radial line profile to construct a matrix of maximum values of $l o g$ likelihood functions as a function of target size. This matrix is displayed graphically in Fig. 3 as a contour plot. Using the log likelihood function as an estimator of target size suggests a target having a $2 \mathrm{ft}$. horizontal extent and an $8 \mathrm{ft}$. vertical extent as compared to the actual $3 \mathrm{ft}$. target diameter. It is clear from this figure that the peak value of $\log$ likelihood function is more sensitive to horizonta! dimension than vertical dimension. Figure 4 displays the values of the $\log$ likelihood function over a vertical cross-section as

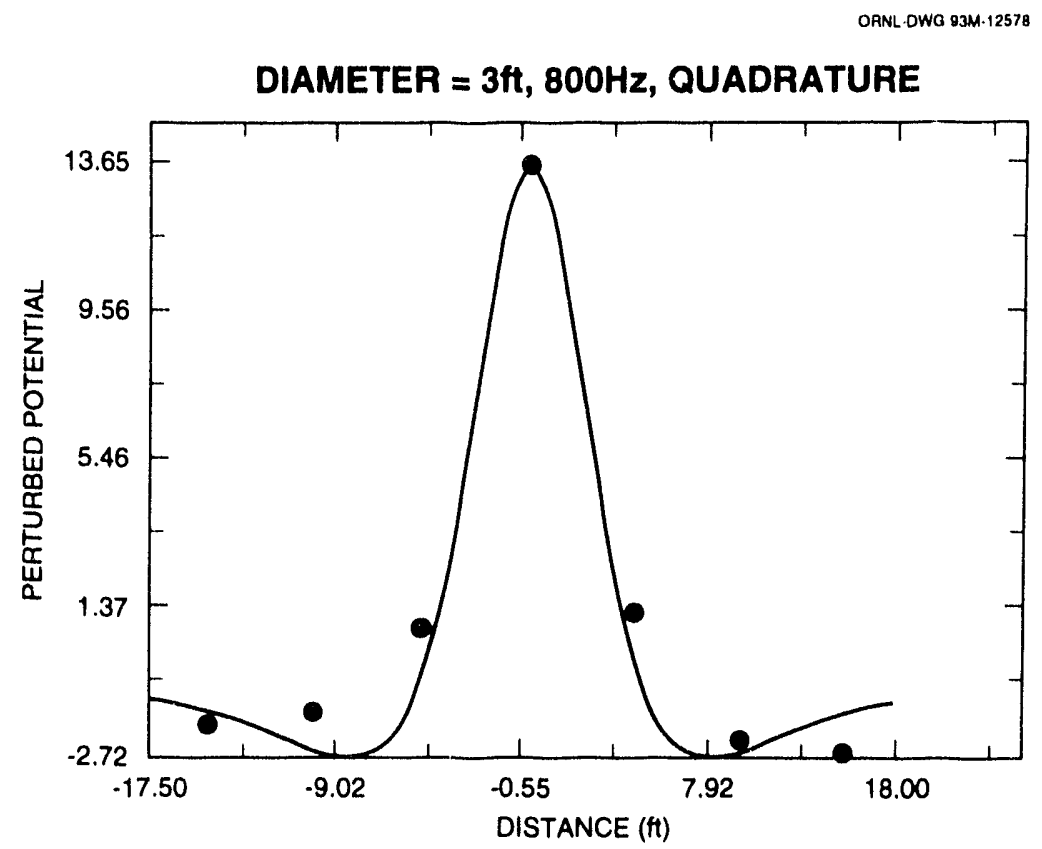

Figure 2: A plot of observed and simulated quadrature EMI data as a function of measurement position over the top of a UST. The simulated results are the solid line and the scaled measured values are the ${ }^{*}$ 's.

gray levels for a $2 \mathrm{ft}$. by $8 \mathrm{ft}$. target. The best estimate of the location of this target is the position of the peak in log likelihood function (the black region). In this case, the target occurs at the correct horizontal position but at a depth of about $6 \mathrm{ft}$. This depth is probably slightly too deep to be correct. Figure (5) is a similar gray level plot but using a rectangular target having a $3 \mathrm{ft}$. horizontal and vertical extent. Using a better estimate of target dimensions yields a correct estimate of the depth to the center of the target of about $4 \mathrm{ft}$. Figure (6) is a contour plot similar to Fig. (3) but for the data as scaled in Figs. (1) and (2). In this case, the best estimate for the target size is a 2 ft. square, a much better estimate than that shown in Fig. (3), with the contours more tightly clustered around this maximum value than for the unscaled data. The major 
difference between the scaled and unscaled data is the significant discrepancy between the peak value of in-phase and quadrature components that has been removed by the scaling. This fact suggests this discrepancy as the cause for poor performance of the maximum likelihood estimator in target size estimation.

The same analysis procedure used for target size and location estimation applied to the radial data was used for data acquired along a perpendicular line over the top of the long axis of the UST. This is subsequently referred to as the axial profile. Figure 7 is a contour plot demonstrating target dimension sensitivity. The proper target dimensions are approximately $8 \mathrm{ft}$. horizontally and $3 \mathrm{ft}$. vertically; however, the parameter search reveals a best estimate of $10 \mathrm{ft}$. square, with a broad area of relatively high maximum log likelihoods over the entire computed range of vertical extents for a horizontal extent of $6 \mathrm{ft}$. Figure 8 is a gray scale plot of spatial variations of log likelihood for a $10 \mathrm{ft}$. square target. Figure 9 is a similar plot but for the better target size estimate of $8 \mathrm{ft}$. horizontally and $3 \mathrm{ft}$. vertically. For both target sizes, the estimate of horizontal target

ORNL.DWG 93M.12584

Figure 3: Contours of constant maximum log likelihood function as a function of target dimensions for the $800 \mathrm{~Hz}$. radial profile.

location is correct but the depth, at $7 \mathrm{ft}$., is too deep.

It is not clear why the maximum likelihood estimator is relatively poor at estimating target dimensions. It is obvious that the treatment of the target as two-dimensional is questionable. This becomes apparent when considering a measurement line that is close to but not directly over a target. Within the framewurk of a two-dimensional analysis, there is no target within the cross-section and, consequently, the data template model will yield no perturbed potential. In reality, by virtue of the proximity of the measurement to 


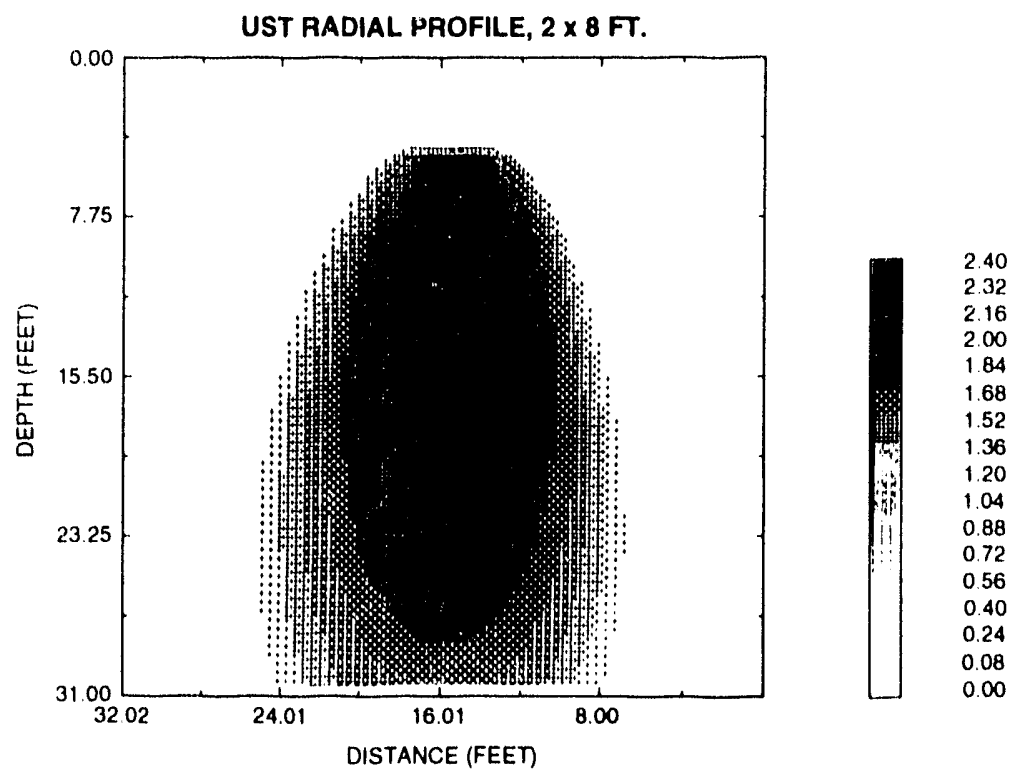

Figure 4: Plot of the spatial variations in log likelihood function, displayed as gray levels, for the $800 \mathrm{~Hz}$. UST radial profile.

ORNL-DWG $93-6251$
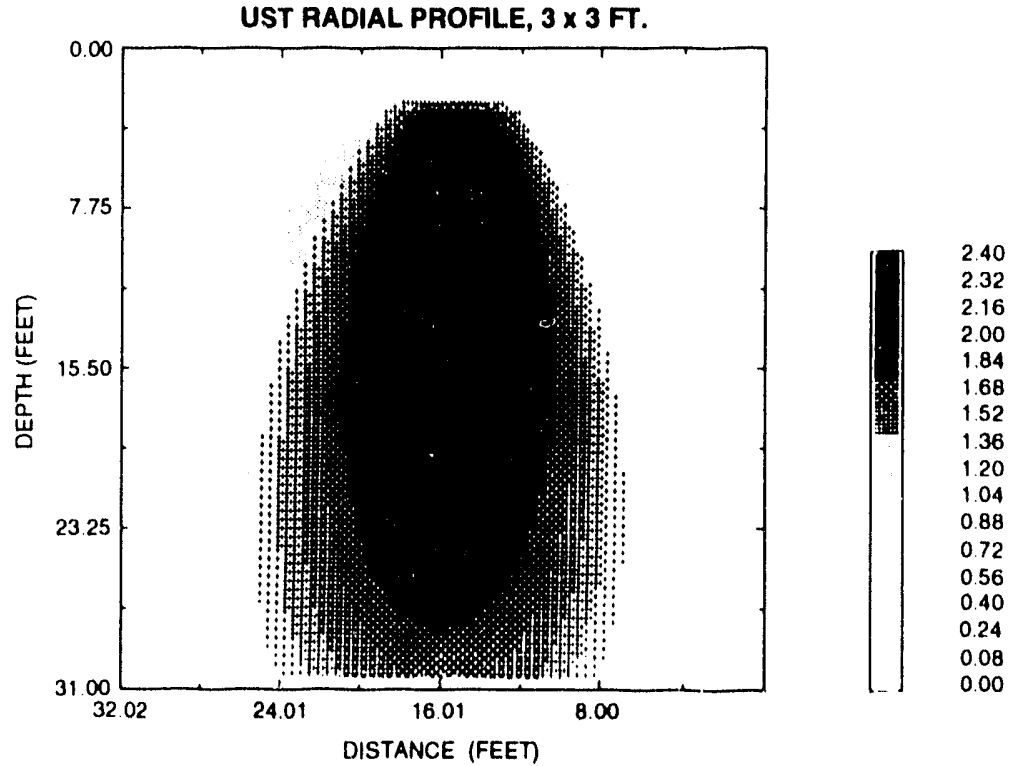

agure 5: Plot of the spatial variations in log likelihood function, displayed as gray levels, for the scaled $300 \mathrm{~Hz}$. UST radial profile. 


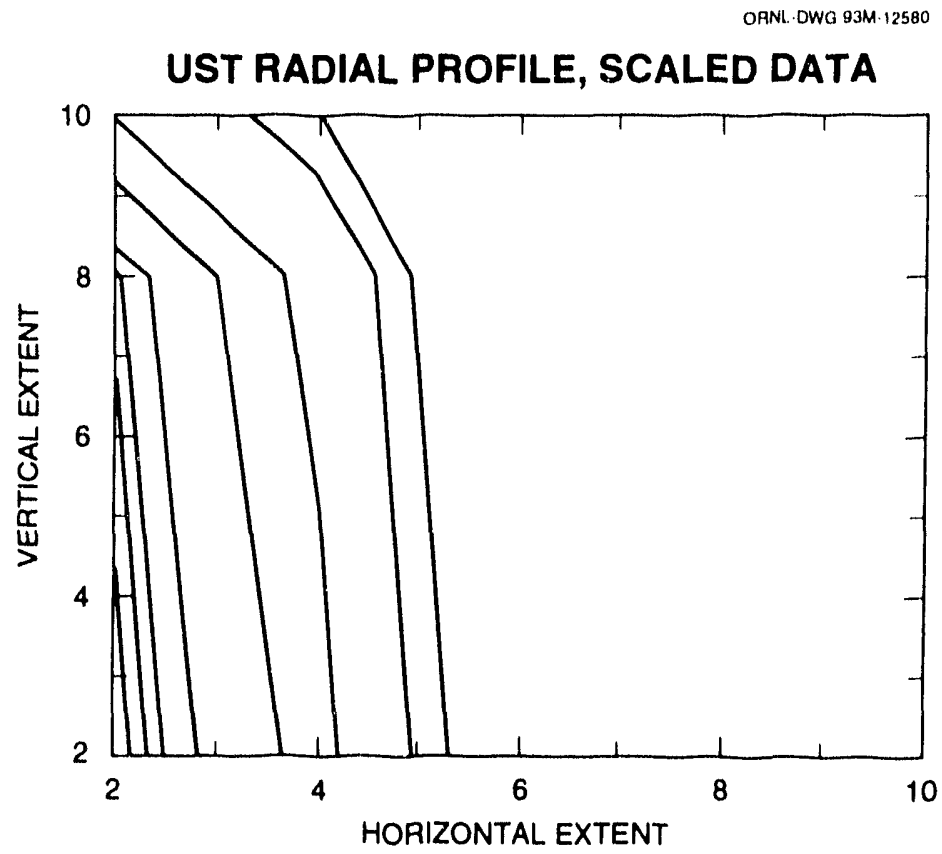

Figure 6: Contours of constant maximum log likelihood function as a function of target dimension for the scaled $800 \mathrm{~Hz}$. radial profile.

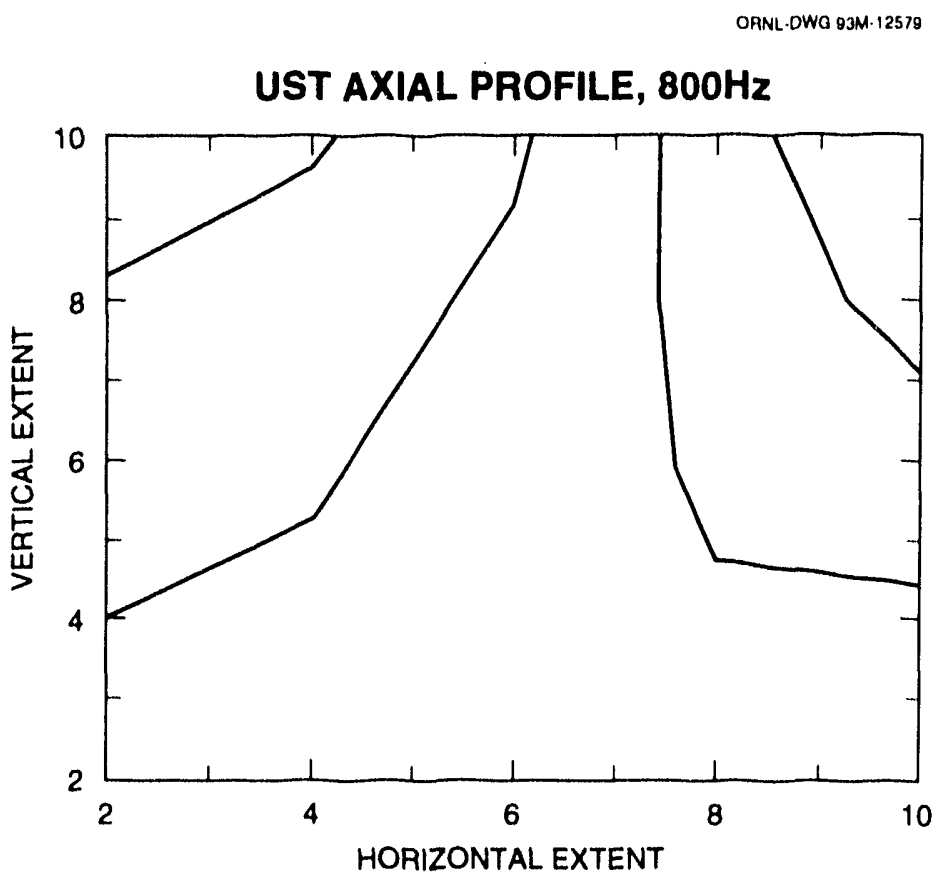

Figure 7: Contours of constant maximum log likelihood function as a function of target dimensions for the $800 \mathrm{~Hz}$. axial profile. 


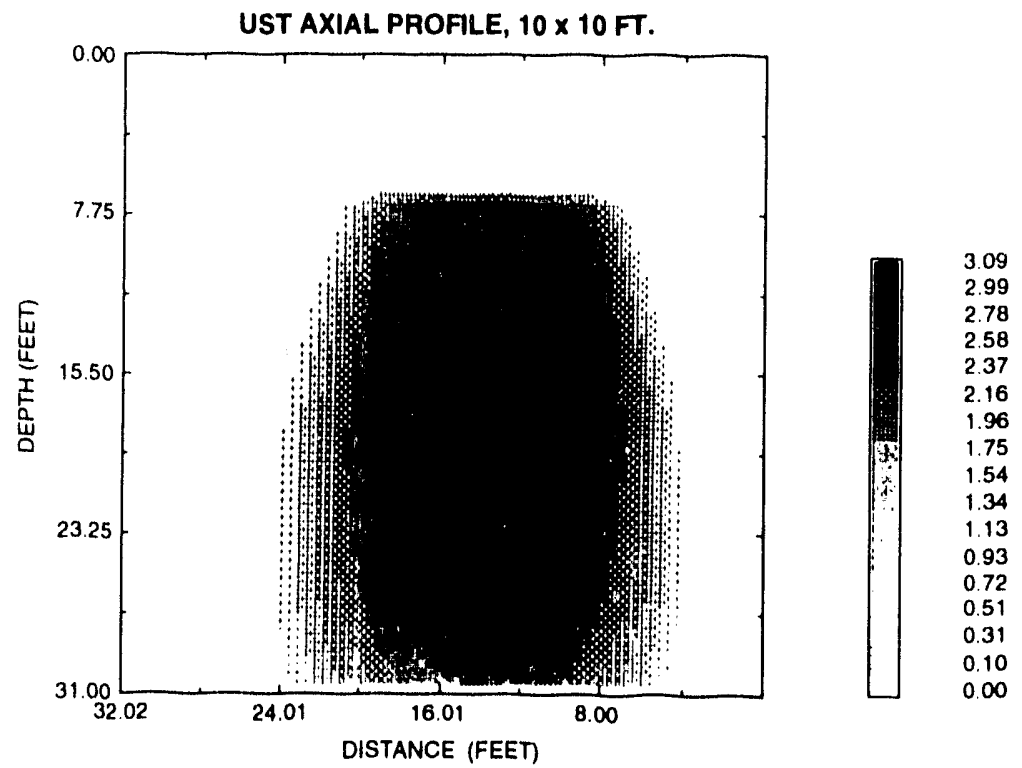

Figure 8: Plot of the spatial variations in log likelihood function for the axial profile and a $10 \mathrm{ft}$. by $10 \mathrm{ft}$. target.

\section{ORNL-DWG 93-6250}

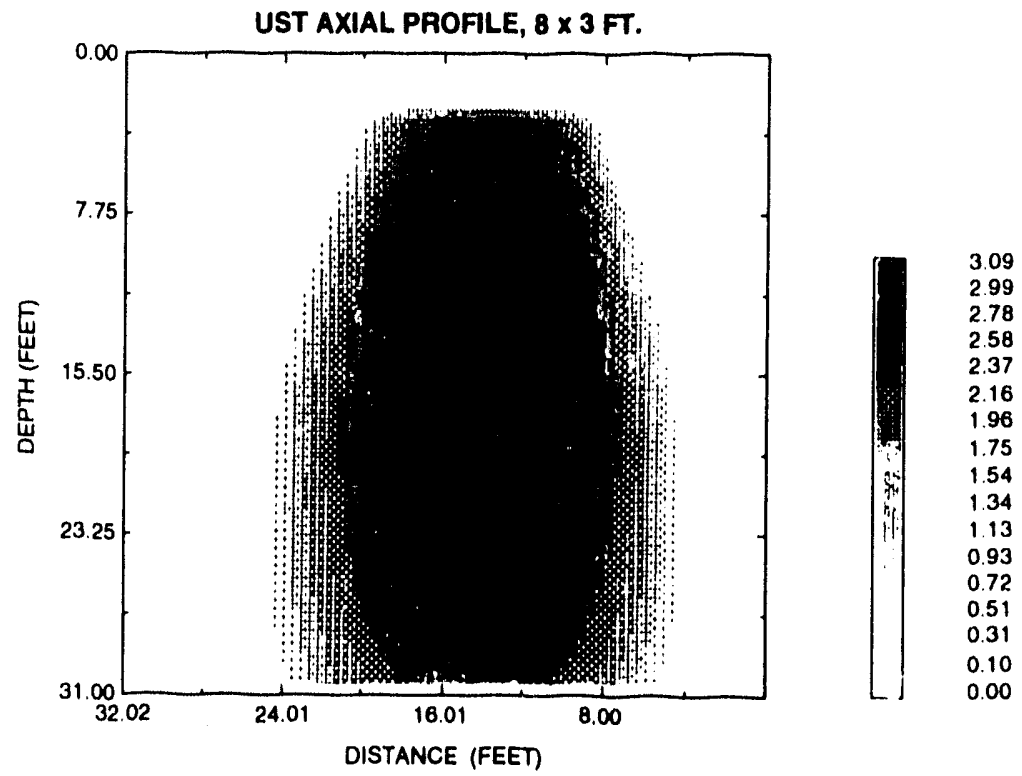

Figure 9: Plot of the spatial variations in log likelihood function for the axial profile and an $8 \mathrm{ft}$. by $3 \mathrm{ft}$ target. 
the target, an actual perturbed potential would be measurement. Target dimensionality will be considered in somewhat more detail in the subsequent subsection. Target shape is another factor impacting the performance of the maximum likelihood estimator. The UST was treated as orthogonal two-dimensional rectangular targets. While this might be a reasonable approximation for a cylinder, the US'T has several filler pipes extending up to the ground surface at one end. This more complex shape was not represented in the data templates and the estimated $10 \mathrm{ft}$. vertical extent in the axial profile no doubt is a manifestation of the presence of these pipes.

A third factor influencing the resolution capacity of the maximum likelihood estimator is operating frequency. It is expected that resolution will be frequency dependent with increased resolution offered by increased operating frequency. The $800 \mathrm{~Hz}$. operating frequency used in the analysis to this point is the low end of the normal operating range. A search over target dimensions for an $9600 \mathrm{~Hz}$. operating frequency provided target dimension estimates of 0 . $\mathrm{ft}$. horizontal by $2.0 \mathrm{ft}$. vertical for the radial profile, and $11.5 \mathrm{ft}$. horizontal $2.6 \mathrm{ft}$. rentical for the axial profile. This size estimate is considerably improved for the axial profile and the target location estimate is correct.

\subsection{Target Dimensionality}

It is beyond the scope of this study to develop a three-dimensional target data template model for EMI for use in quantifying the inaccuracies of treating a target that is actually three-dimensional as two-dimensional. A qualitative understanding of the situation can be obtained by considering a similar evaluation using low frequency propagating waves (radar) simulations. Consider radar measurements made along a line on the ground surface above a buried spherical inclusion having a radius smaller than the wavelength. Simulated data for such a configuration is given in Fig. (10). Treating this target as two-dimensional defined by the intersection of the sphere with the vertical cross-section below the measurement line yields a circular target having the same radius as the sphere. Figure 11 displays simulated radar data for this two-dimensional target. The major differences between the two- and three-dimensional results are that the main lobe of the two-dimensional data is narrower than that for the three-dimensional data and the threedimensional data exhibits two side lobes which are absent in the two-dimensional data. If such a side lobed structure appeared in EMI measurements, it is likely that they would not be resolved by a five foot measurement spacing as used in the field measurements addressed in Sects. 3.1 and 3.2. Assuming this to be the case, apparent differences between a circular and spherical target would be a single lobed distribution exhibiting a slight broadening of the base as compared to data from a two-dimensional target. Although such differences are subtle, they are sufficient to manifest a significant change in the result obtained from the maximum likelihood estimator. 


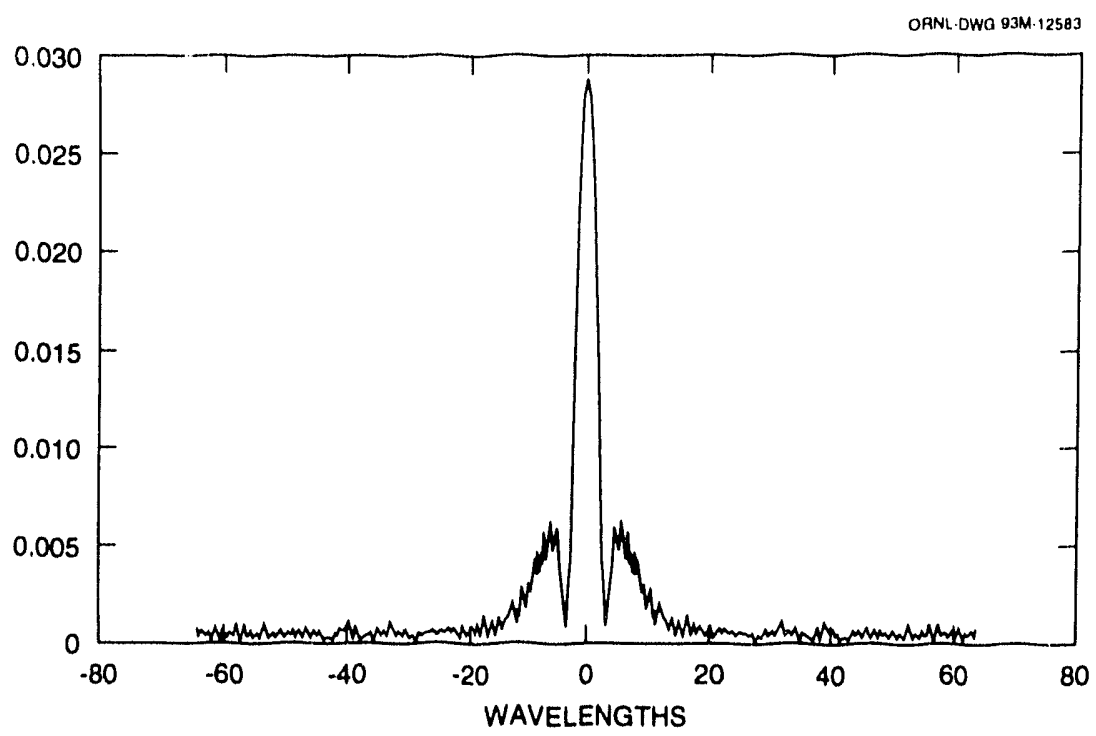

Figure 10: Plot of simulated backscattered data along a line over the top of a spherical target.

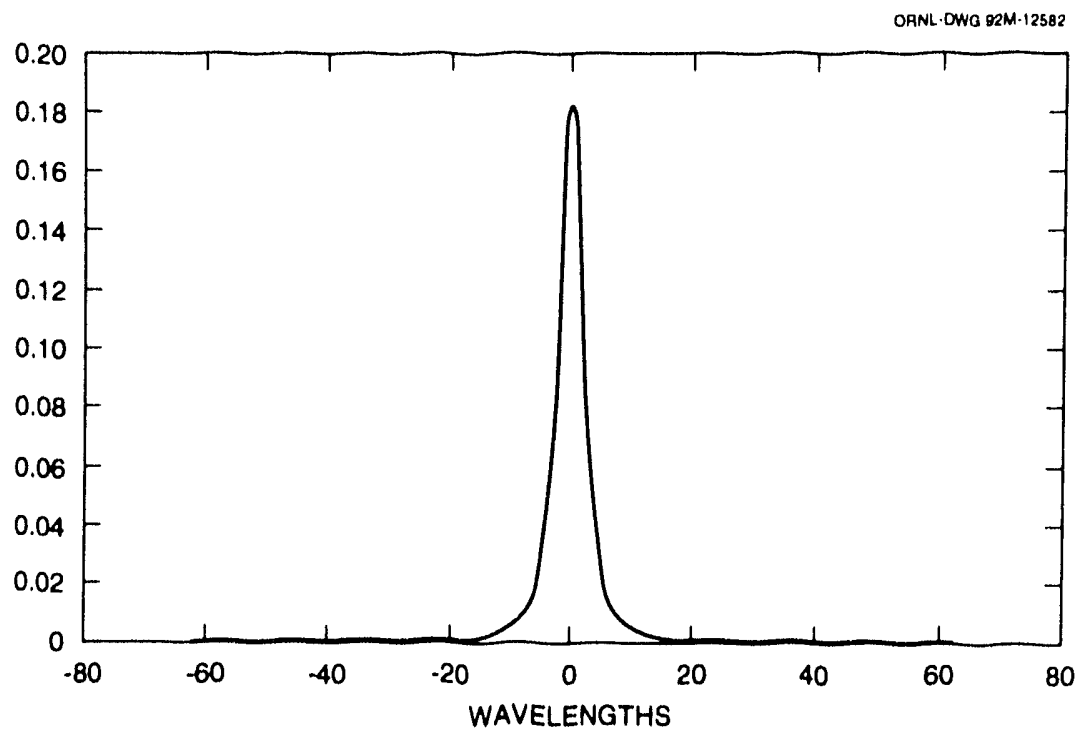

Figure 11: Plot of simulated backscattered data along a line over the top of circular target. 


\section{The Field Study}

A field study was conducted on the Magnetic Test Range of the Naval EOD Tech Center at Indian Head, Maryland. This range contains inert ordnance of various sizes buried with different orientations and at different depths. A $200 \mathrm{ft}$. by $200 \mathrm{ft}$. portion of this range was selected for study and a coordinate system was established having its origin at the southwest corner with orthogonal axes extending to the north and east. The main geophysical tools selected for use at this site were a flux gate gradient magnetometer and Geophex's GEM 1 EMI tool. The flux gate magnetometer was selected because, by virtue of the gradient measurement, it does not respond to temporal variations in the background magnetic field and because it was recently employed quite successfully at a buried unexploded ordnance site in Los Alamos, New Mexico. The GEM 1 tool was selected because of its established high sensitivity and its ability to employ multiple operating frequencies. In addition to these primary tools, a Geonics EM 31 EMI tool that operates at a fixed frequency of $9800 \mathrm{~Hz}$. and a proton precession magnetometer were available for use at the site.

Measurements were made with both the GEM 1 and flux gate magnetometer at five foot intervals over the entire study area. With the GEM 1, operating frequencies of $800,1280,1600,3200,4800,6400$, and $9600 \mathrm{~Hz}$. were used. These two tools were used simultaneously; however, the EM 31 was only used to randomly survey selected portions of this area. The reason for this is that cross contamination of data can result from primary coils of multiple EMI tools in close proximity. No significant anomalies were found with either the flux gate magnetometer or the EM 31. The possible reasons for the failure of the EM 31 is not clear; however, the failure of the magnetometer appears to be the preset sensitivity set within the tool. The flux gate magnetometer is a commercial one modified to include an analog-to-digital converter and digital display. The magnetometer, itself, should have sufficient sensitivity to detect the objects buried at the test site; however, hard wired within the instrument is a selection of bits to be displayed. The particular choice used at Indian Head was that used previously with good success at Los Alamos. It was apparent early on in this field study that this selection was suboptimal for this particular site but change was impossible without instrument modification. As a result of this experience, the flux gate magnetometer has been modified to allow the user to select, by means of external switches, a desired sensitivity.

As a result of the limitations of the flux gate magnetc neter, a reduced portion of the study area was surveyed with the proton precession magnetometer at the end of the field study. Time constraints prevented a complete investigation of the area with this tool so that only about the northern most half of the area could be surveyed. A plot of constant perturbations in the total magnetic field from the proton precession magnetometer is provided in Fig. 12. A total of eight magnetic anomalies of various strengths appear in the surveyed region and the coordinates of these are given in Table 1. An additional anomaly lies just beyond the survey boundary at about $95 \mathrm{ft}$. east and $205 \mathrm{ft}$. north.

Similar contours for in-phase and quadrature measurements for the seven GEM 1 


\begin{tabular}{|c||c|c|}
\hline Target No. & East (ft.) & North (ft.) \\
\hline 1 & 80 & 150 \\
2 & 75 & 95 \\
3 & 95 & 185 \\
4 & 110 & 180 \\
5 & 130 & 190 \\
6 & 150 & 160 \\
7 & 120 & 90 \\
8 & 125 & 135 \\
\hline
\end{tabular}

Table 1: Locations of magnetic anomalies.

operating frequencies are given in Figs. 13 through 26. Inspection of these figures reveals little correlation of perturbed potential between in-phase and quadrature components at the same frequency or among individual components at different operating frequencies. There are only isolated instanced where weak EMI anomalies occur at the same locations as magnetic anomalies (Fig. 12).

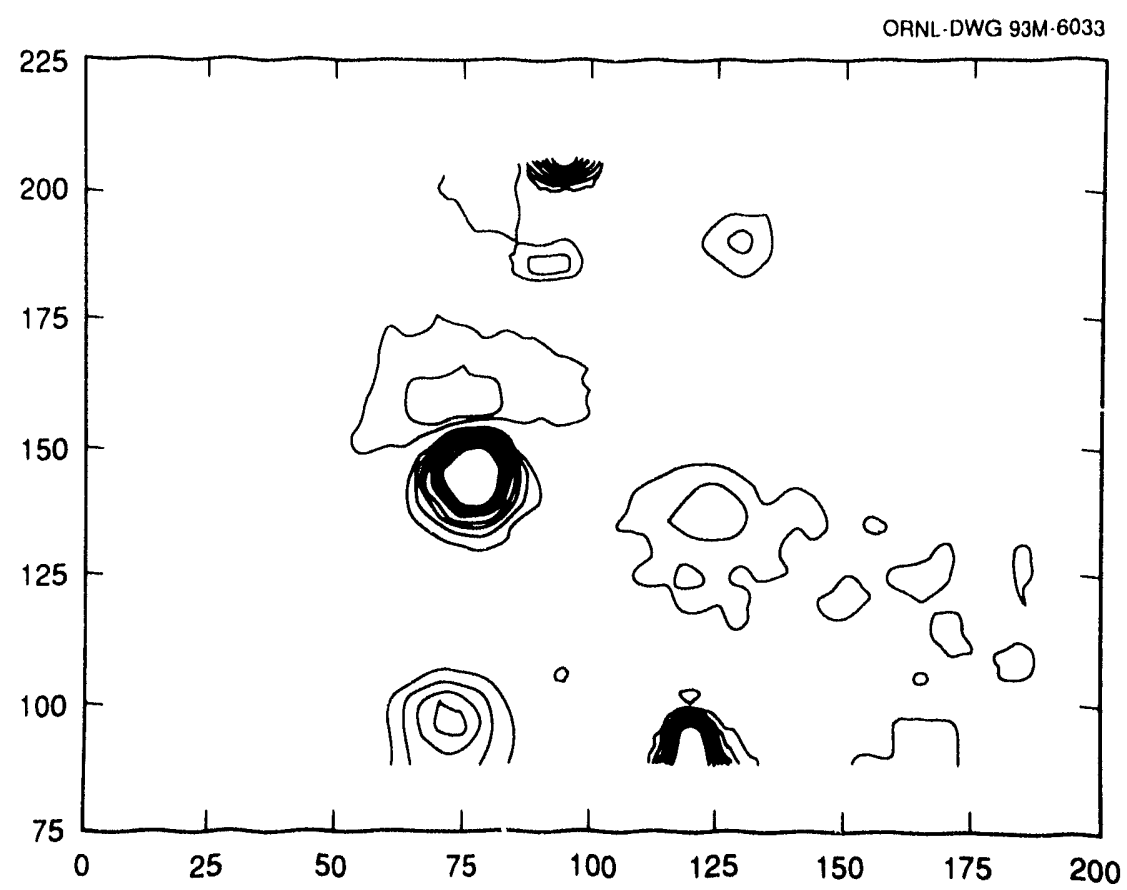

Figure 12: Contour plot of perturbations in total magnetic field from a proton precession magnetometer. 

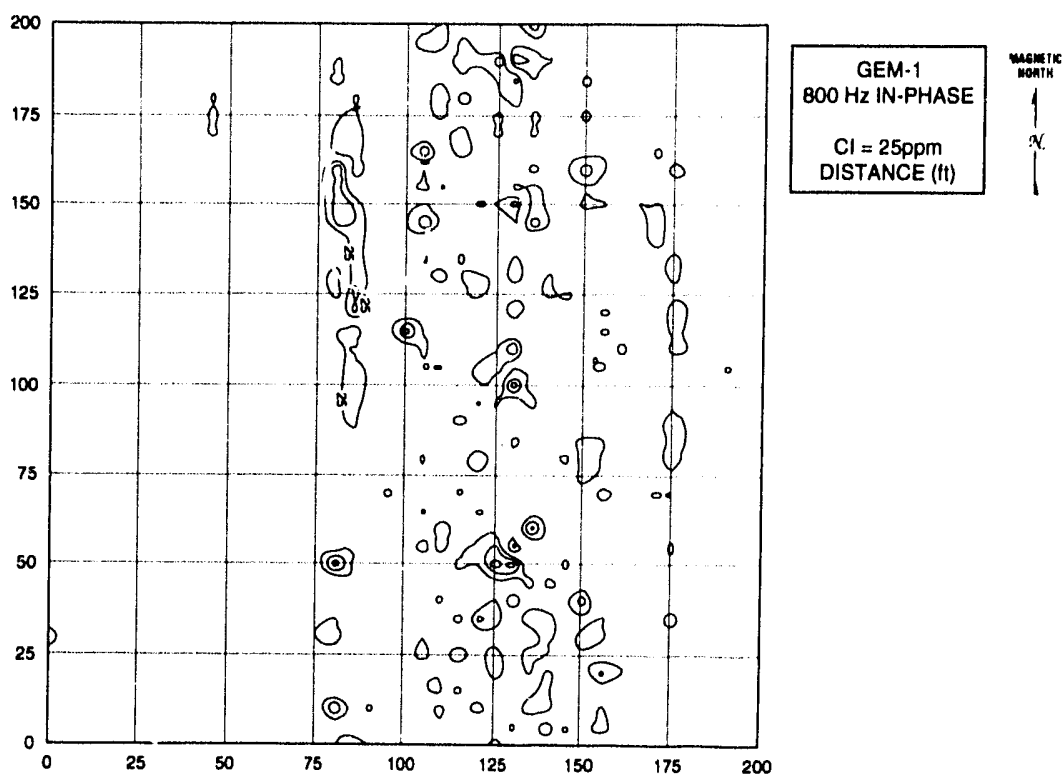

Figure 13: Contours of in-phase component at $800 \mathrm{~Hz}$.
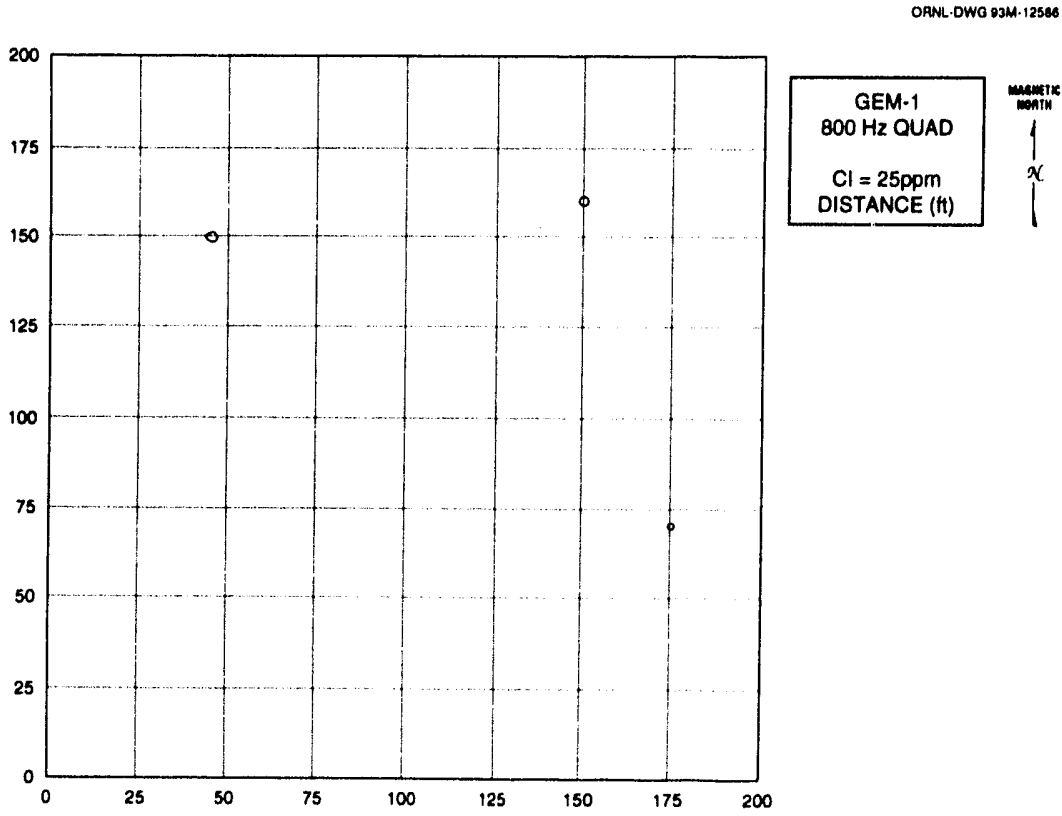

Figure 14: Contours of quadrature component at $800 \mathrm{~Hz}$. 


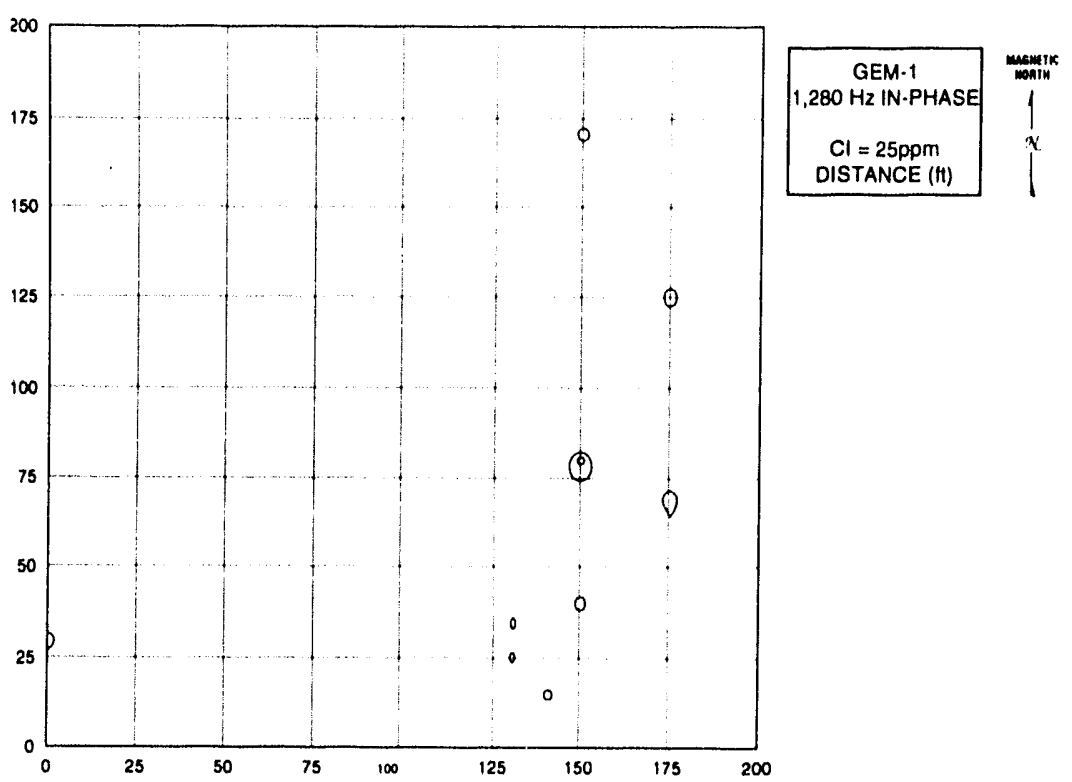

Figure 15: Contours of in-phase component at $1280 \mathrm{~Hz}$.

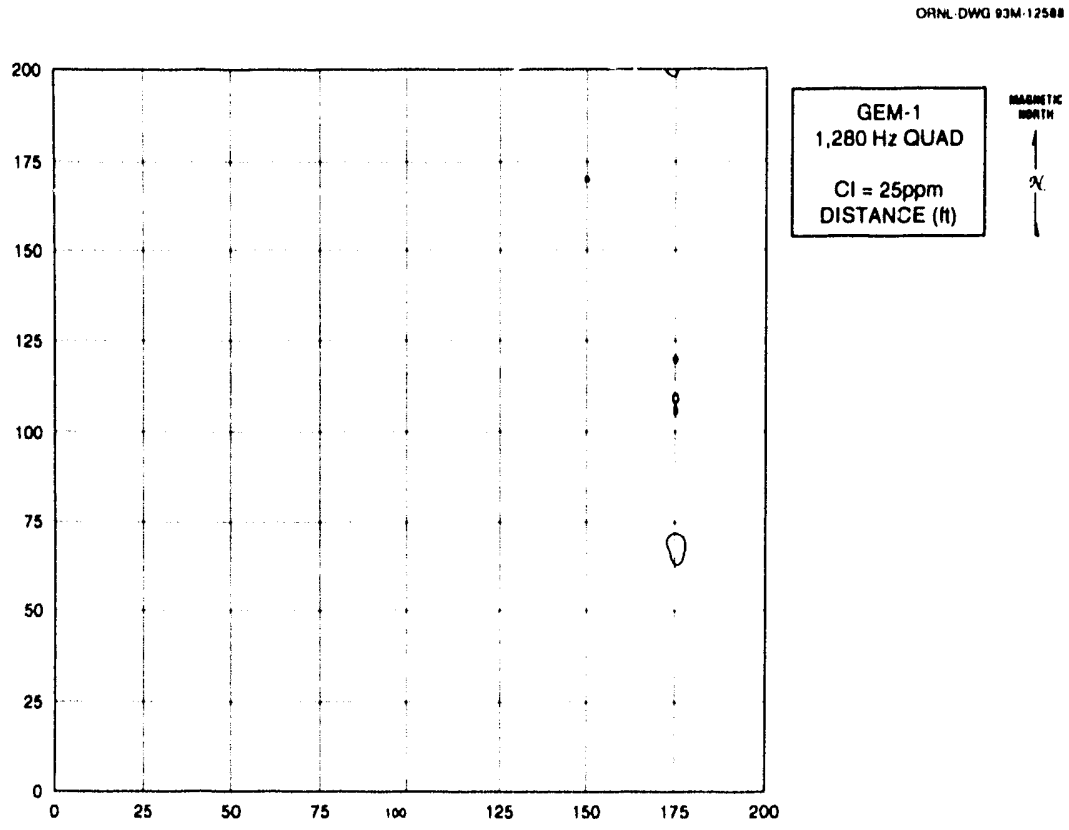

Figure 16: Contours of quadrature component at $1280 \mathrm{~Hz}$. 
ORNL DWG 03M. 12580

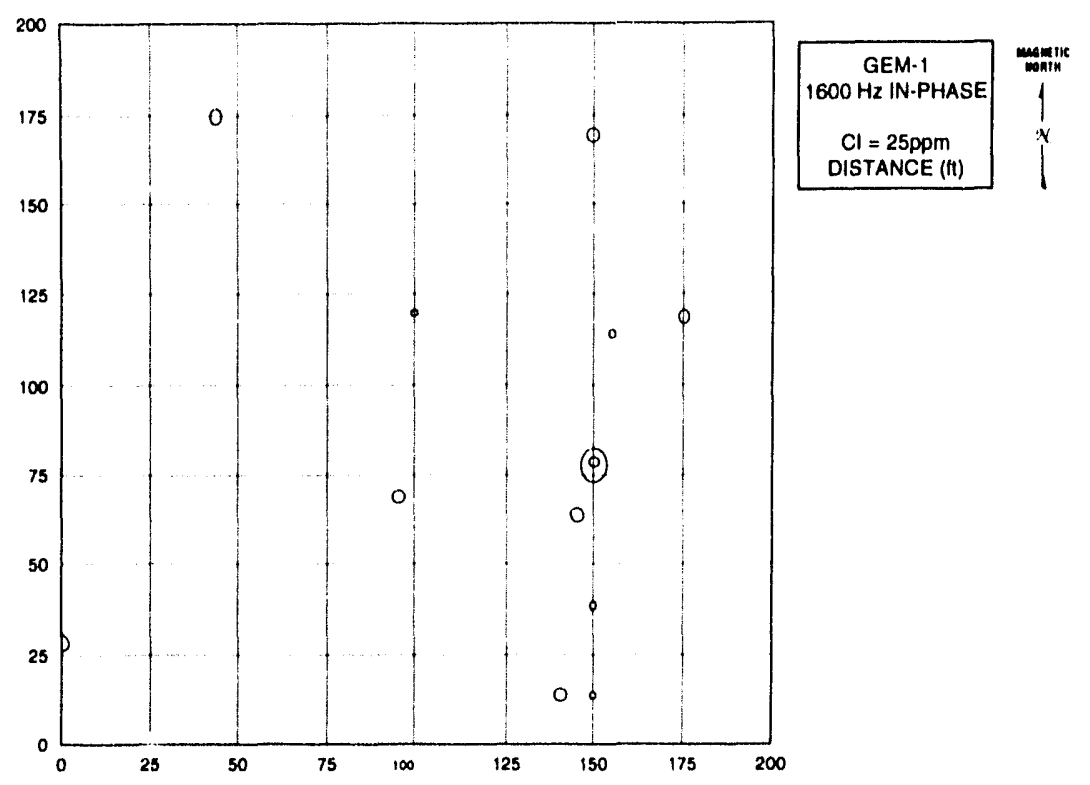

Figure 17: Contours of in-phase component at $1600 \mathrm{~Hz}$.

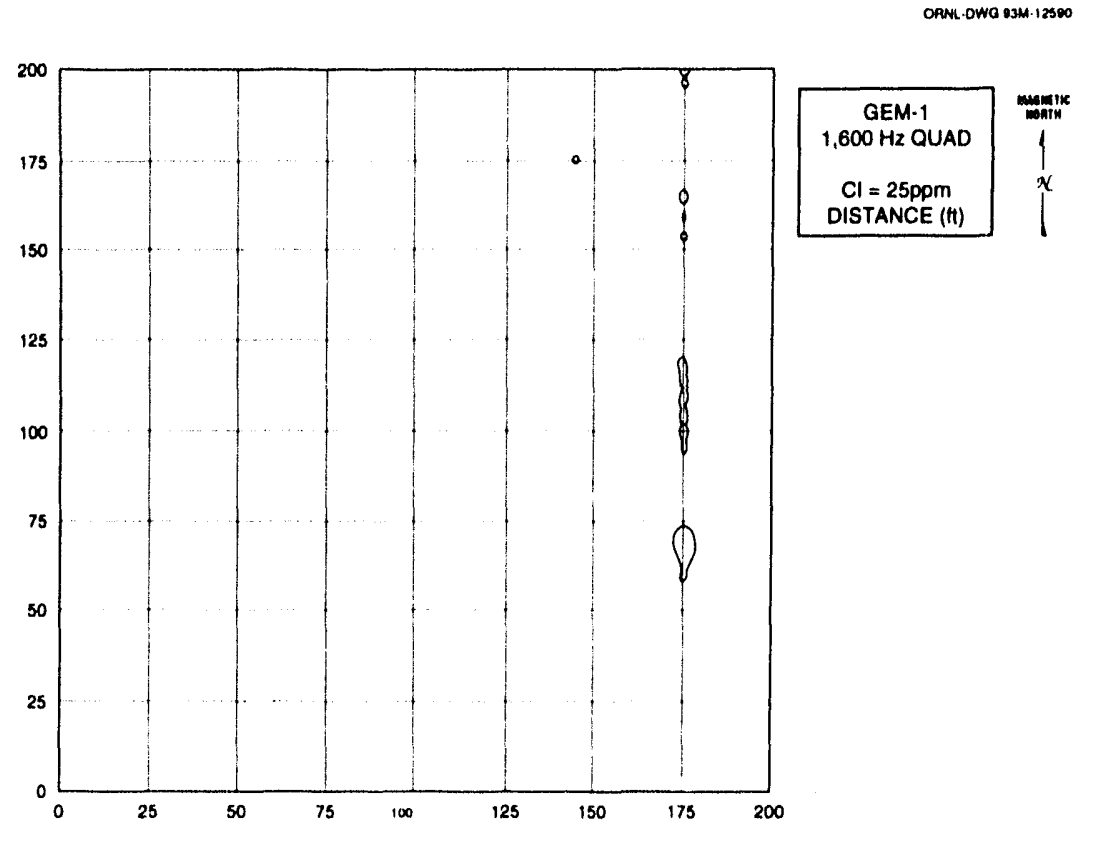

Figure 18: Contours of quadrature component at $1600 \mathrm{~Hz}$. 

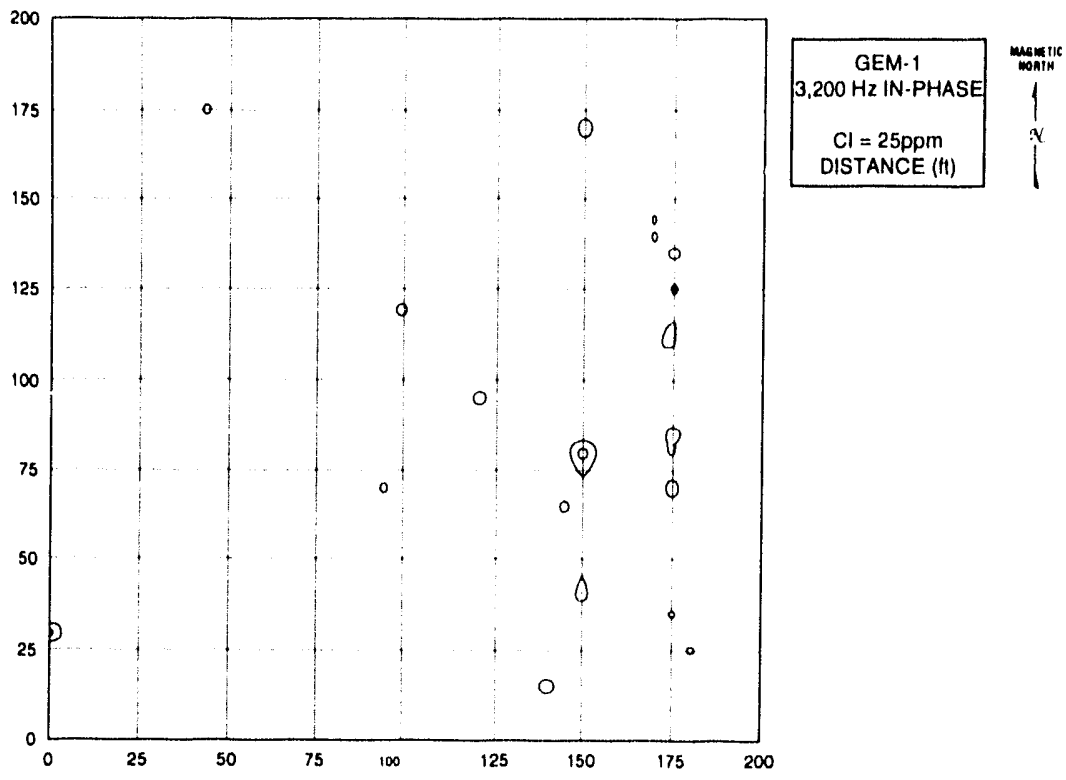

Figure 19: Contours of in-phase component at $3200 \mathrm{~Hz}$.

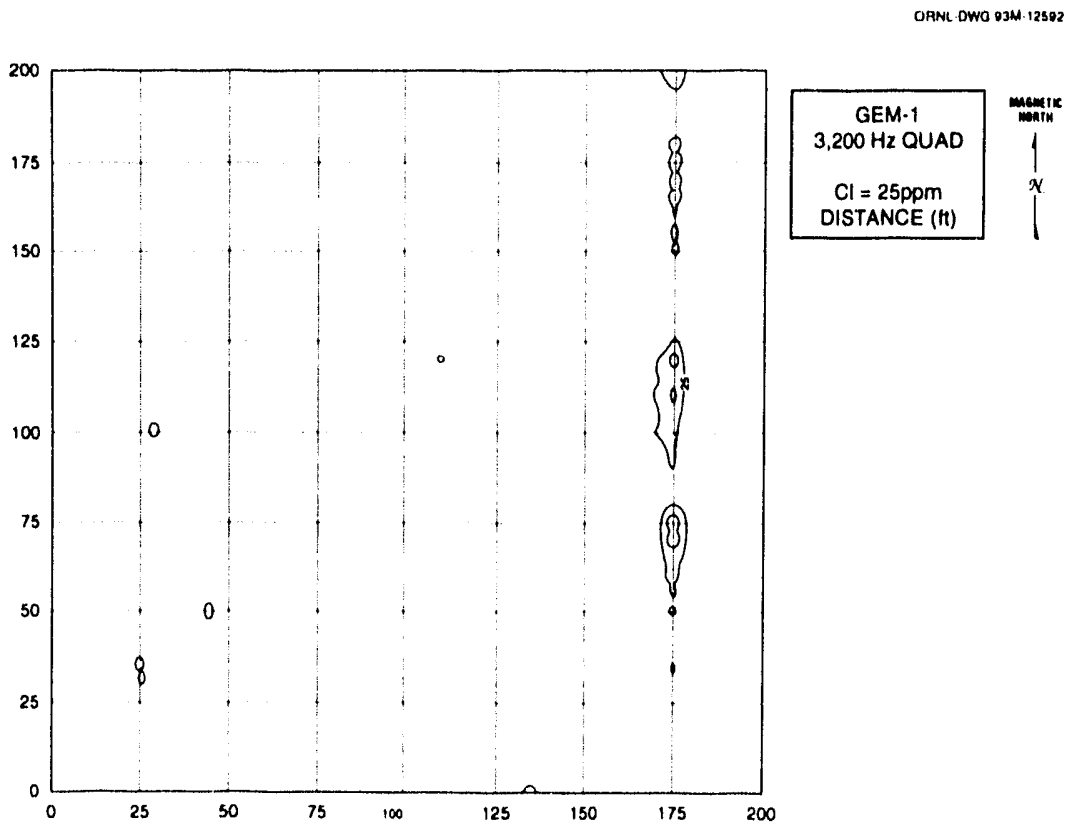

Figure 20: Contours of quadrature component at $3200 \mathrm{~Hz}$. 


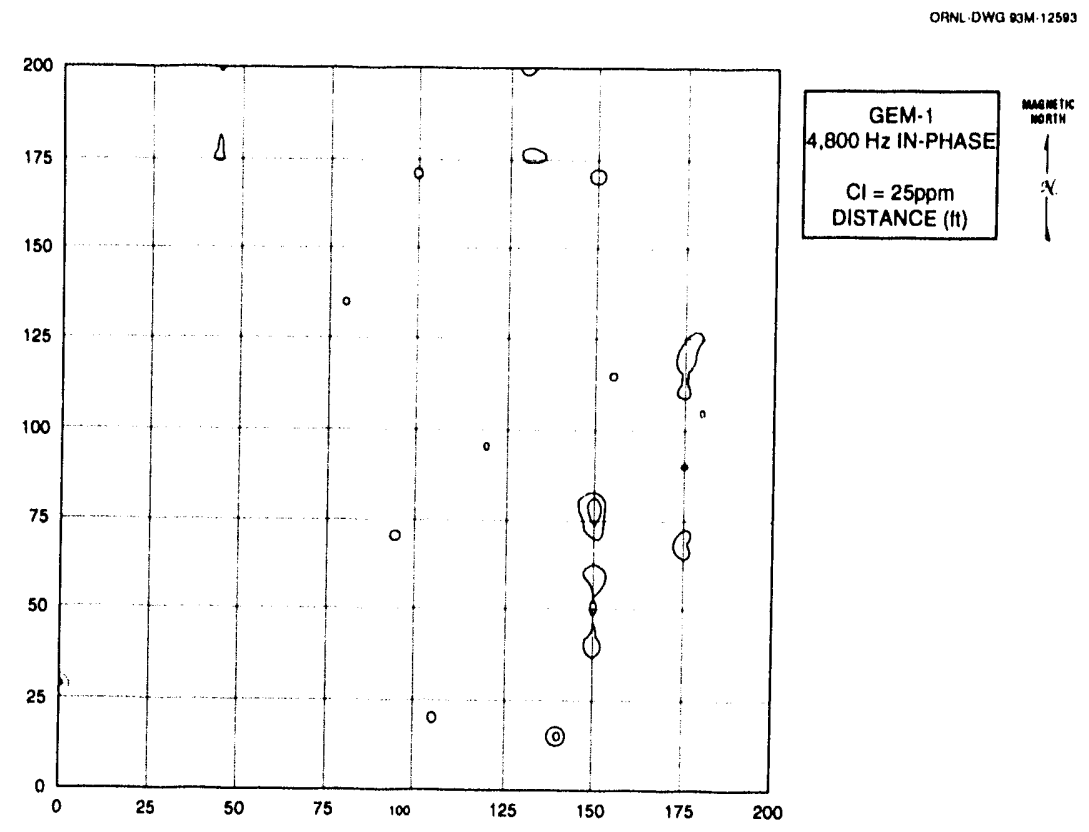

Figure 21: Contours of in-phase component at $4800 \mathrm{~Hz}$.

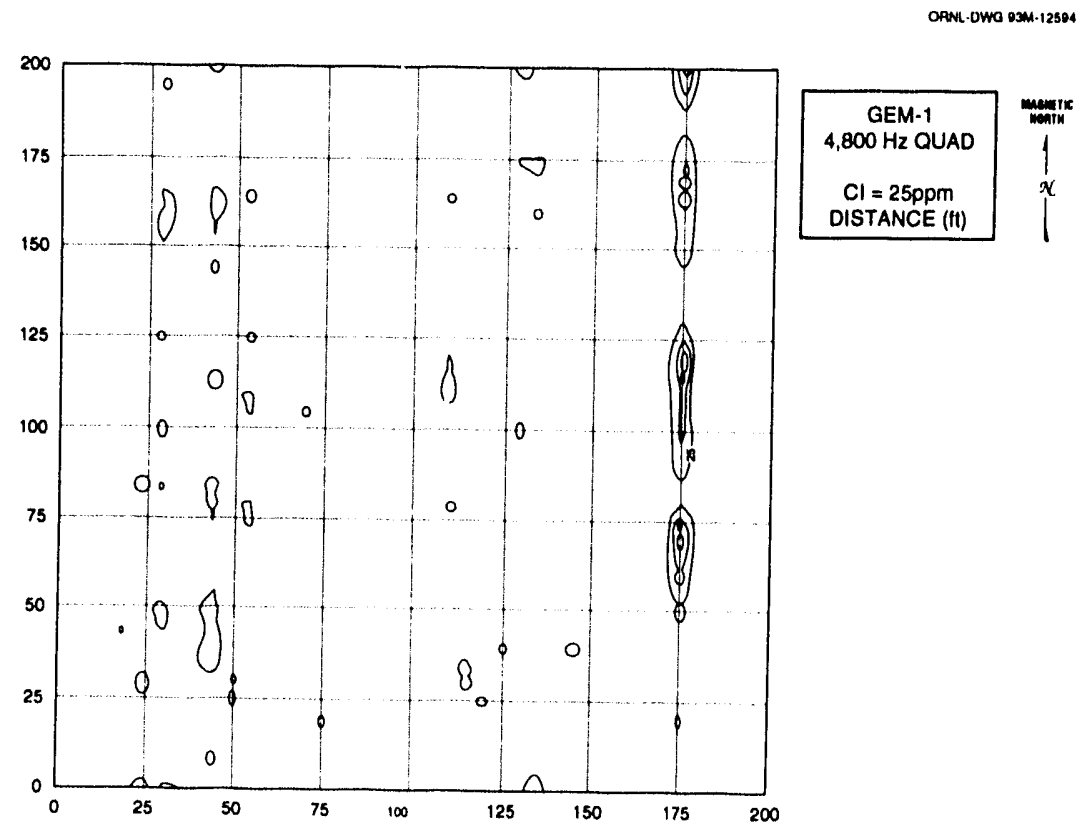

Figure 22: Contours of quadrature component at $4800 \mathrm{~Hz}$. 
ORN. OWG OJM I2505

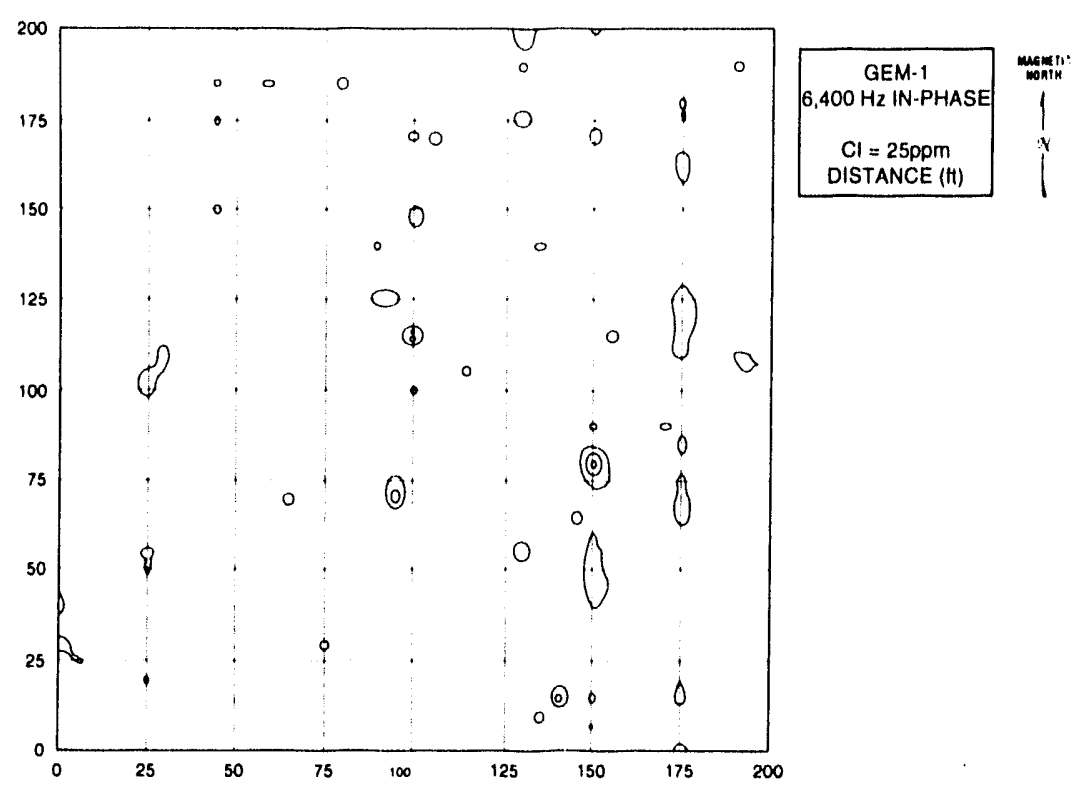

Figure 23: Contours of in-phase component at $6400 \mathrm{~Hz}$.

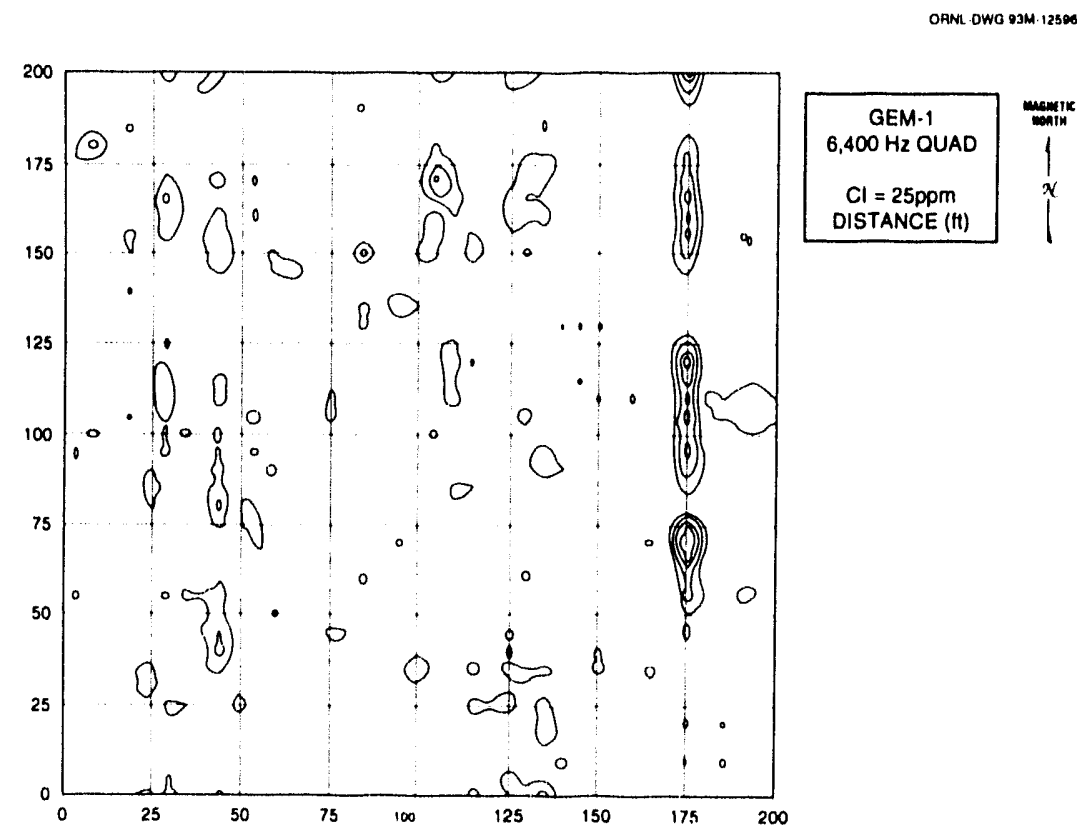

Figure 24: Contours of quadrature component at $6400 \mathrm{~Hz}$. 


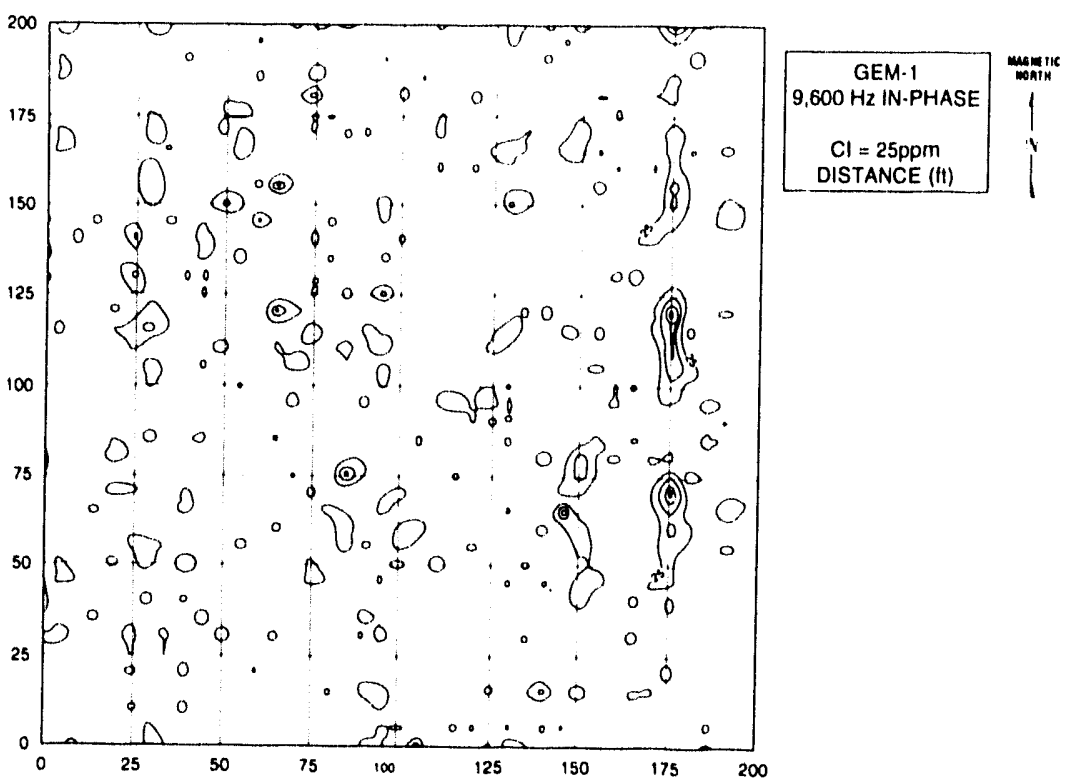

Figure 25: Contours of in-phase component at $9600 \mathrm{~Hz}$.

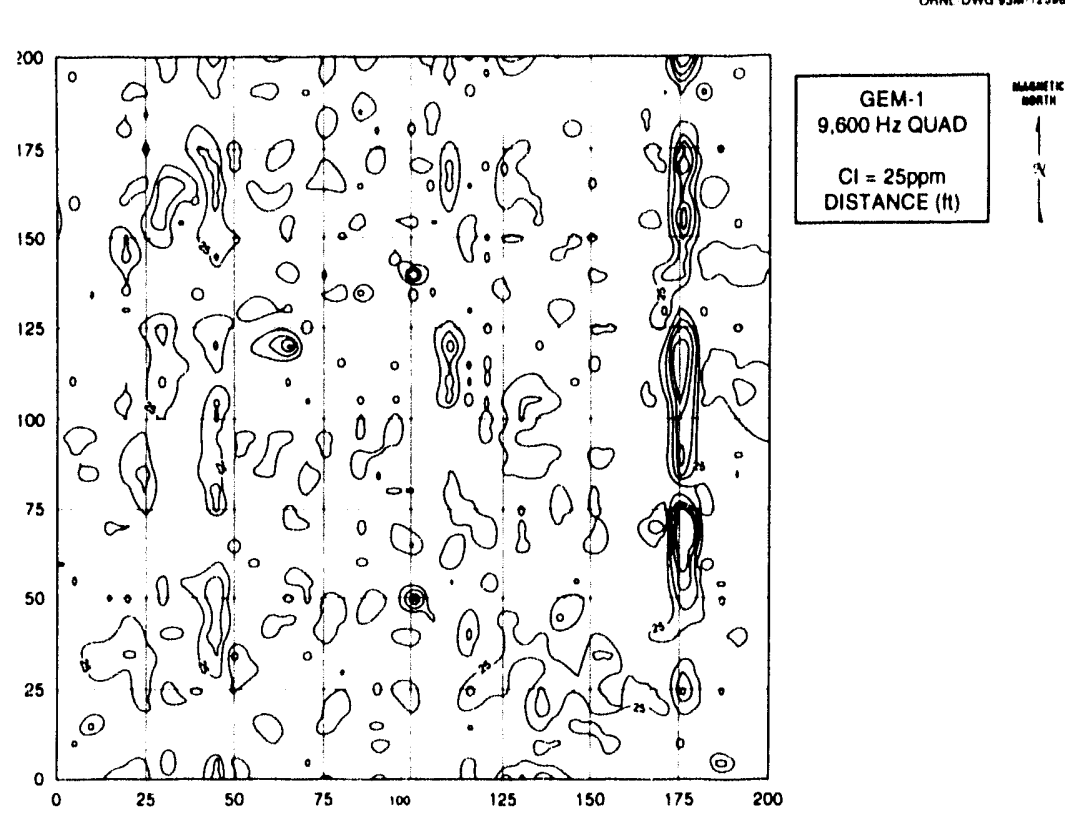

Figure 26: Contours of quadrature component at $9600 \mathrm{~Hz}$. 


\section{Results}

Data acquired with the proton precession magnetometer and the GEM I EMI tool were processed with the maximum likelihood estimator and appropriate two-dimensional data templates derived in Sect. 2. Orthogonal north-south and east-west survey lines intersecting over the approximate tops of magnetic anomalies identified in Table 1 were defined and appropriate segments of these data sets were input into the detection and location algorithms. Signals were processed for all magnetic anomalies; however, some targets were too close to a measurement boundary to allow the application of the signal processing algorithm in one of the directions. EMI data acquired only over two of the magnetic anomalies were processed. For both the magnetometer and EMI data, a search over rectangular target size was first performed to estimate the size of individual targets and this size was used to compute the spatial distribution of the log likelihood function in order to estimate each target's location.

\subsection{Magnetic Data}

The locations of magnetometer data lines used in the detection and location estimation algorithm are identified in Fig. 27. These lines are identified with a number and an E or $\mathrm{N}$ designation. The number defines the particular anomaly as identified in Table 1 and the $\mathrm{E}$ or $\mathrm{N}$ designates the orientation of the line as either east to west or north to south, respectively. A summary of the funding from this analysis are provided in Table 2. This table gives target position as distances east and north of the origin; target dimensions in the east-west, north-south, and vertical directions, as well as the target depth. Missing entries in this table are a result of the lack of an appropriate survey line. Based on this analysis, it appears that Target 1 is the largest and is oriented vertically. Target 2

\begin{tabular}{|c||c|c|c|c|c|c|}
\hline \multicolumn{1}{|c||}{$\begin{array}{c}\text { Target } \\
\text { Number }\end{array}$} & \multicolumn{2}{c|}{ Target Location (ft.) } & \multicolumn{3}{c|}{ Target Dimensions (ft.) } \\
\cline { 2 - 7 } & East & North & Depth & East-West & North-South & Vertical \\
\hline \hline 1 & 80. & 150. & 8.0 & 3.0 & 0.1 & 5.0 \\
2 & 75. & 95. & 6.0 & - & 3.0 & 0.01 \\
3 & 95. & 185. & 2.0 & 0.1 & 0.2 & 1.0 \\
4 & 110. & 180. & 2.0 & 0.5 & - & 1.5 \\
5 & 130. & 190. & 3.0 & 0.1 & 0.1 & 2.5 \\
6 & 150. & 160. & 2.5 & 0.5 & 0.1 & 1.0 \\
7 & 120. & 90. & 3.0 & - & 0.1 & 2.5 \\
8 & 125. & 135. & 13. & 0.1 & 0.1 & 2.5 \\
\hline
\end{tabular}

Table 2: Summary of the results of the application of a maximum likelihood estimator to magnetometer data. 
also may be large, although one dimension is missing, and has a horizontal orientation. Targets 3, 4, and 6 are the smallest targets, while targets 5, 7 , and 8 are in an intermediate size range. 'largets 1, 5, 7, and 8 appear to have a vertical orientation. The deepest target is 'larget 8 , however, 'Targets 1 and 2 are also relatively deep in comparison to the other targets.

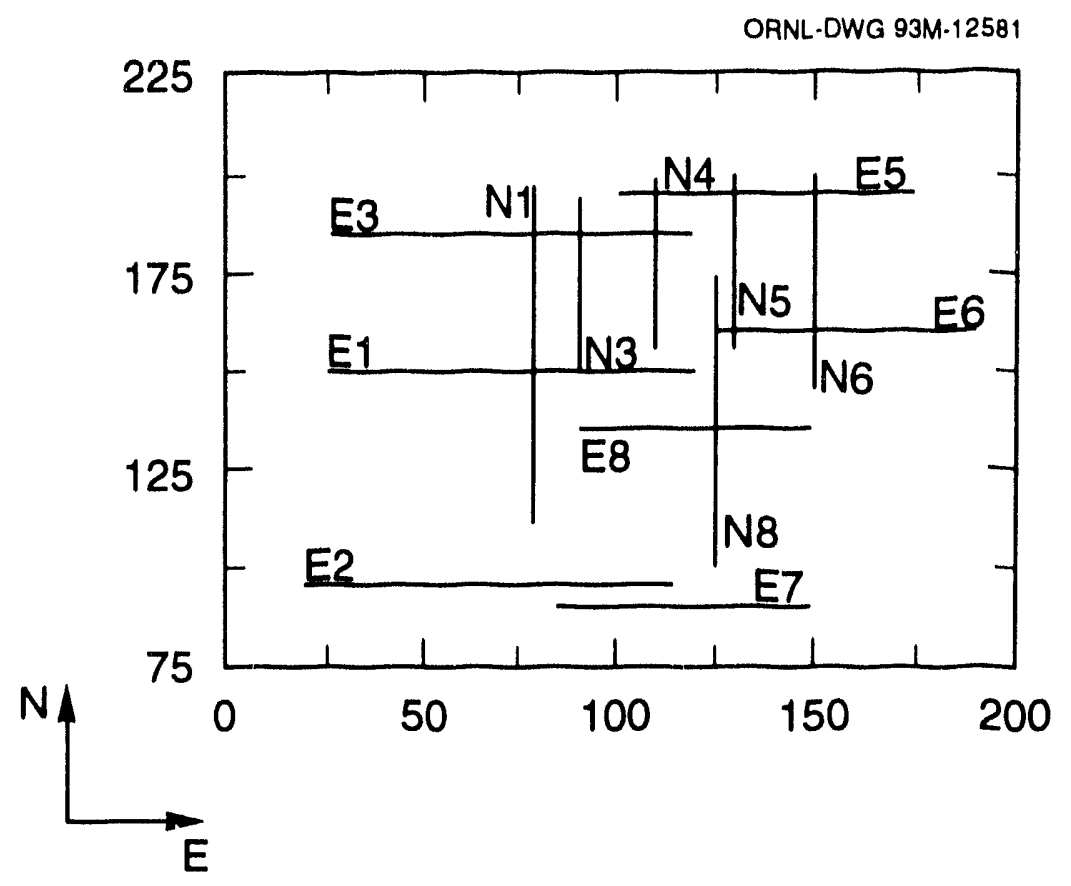

Figure 27: Location of the magnetometer line segments used in signal processing.

Selected gray-scale plots of the spatial variations of the log likelihood function are given in Figs. 28 through 34 . Figure 28 displays the spatial variations in log likelihood function, $F_{L}$, over a vertical cross-section below line E2. A clear peak in $F_{L}$ is evident as the small black area about $8 \mathrm{ft}$. deep and $75 \mathrm{ft}$. east of the origin. Figure 29 is a similar image below line E3. Here the target appears as a large negative value of $F_{L}$, apparent as the white area at a depth of $4 \mathrm{ft}$. and $95 \mathrm{ft}$. east of the origin, suggesting that this target has a magnetic permeability less than background. This "inverted" contrast is surprising since all targets have a ferromagnetic casing that should represent a strong positive contrast. One possible explanation is that this target has some intrinsic magnetism (i.e., it is permanently magnetized) which, when taken together with the perturbation it produces in the Earth's magnetic field yields a negative anomaly. A similar inverted contrast was found for this target along the north-south line with the only difference being that the target depth is $2 \mathrm{ft}$. rather than $4 \mathrm{ft}$.

Figure 30 is a gray scale plot of the spatial variations in $F_{L}$ below survey line N6. This image is included because it shows two anomalies within the same vertical cross-section. The dominant feature occurs in the upper right of this image at a distance north $160 \mathrm{ft}$. and 2.5 feet deep. A much weaker feature appears in the upper left. There is no evidence 


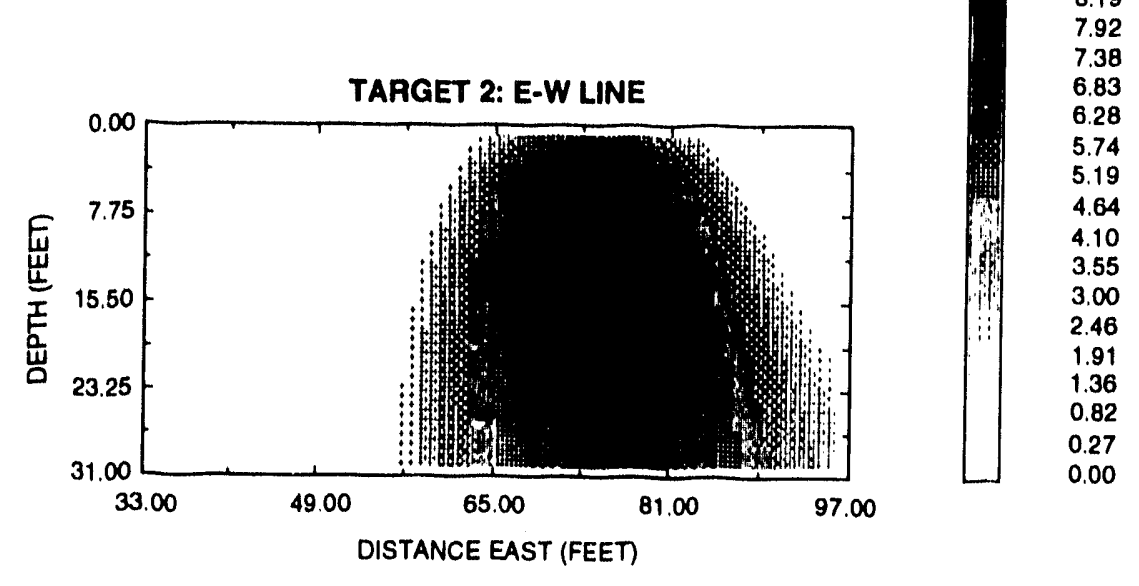

Figure 28: Spatial distribution of the log likelihood function, displayed as gray levels, below magnetometer line E2.

to suggest that this feature is real and it is poorly correlated with the target template. Figures 31 and 32 are gray scale images of the $\log$ likelihood function below lines E6 and ET, respectively. These images are included as companions to similar images, presented in the next subsection, obtained for these target lines using EMI data.

Target 8 occurs in an area where there is no record of any test ordnance burial. Figures 33 and 34 provide gray scale images of $F_{L}$ over vertical cross-sections through this target in a east-west and north-south direction, respectively. These images clearly show a quite strong match to a data template strongly suggesting that this is a real subsurface feature.

\subsection{EMI Data}

Since no definitive anomalies were evident in the EMI data, it was assumed that all buried features of interest were identified by proton precession magnetometer (Fig. 12) with locations given in Table 1. The EMI data sets acquired with the GEM 1 (Figs. 13-26) were visually inspected in an attempt to identify any weak EMI anomalies in the vicinity of established magnetometer anomalies. This search revealed very weak EMI anomalies at Target 6, for operating frequencies of 1280 and $1600 \mathrm{~Hz}$, and at Target 7 , for 4800 and $9600 \mathrm{~Hz}$. The maximum likelihood estimator was applied to EMI data at these locations for the relevant frequencies. The signal processing was far more successful for east-west lines than for north-south lines. The reason for this might be that the GEM 

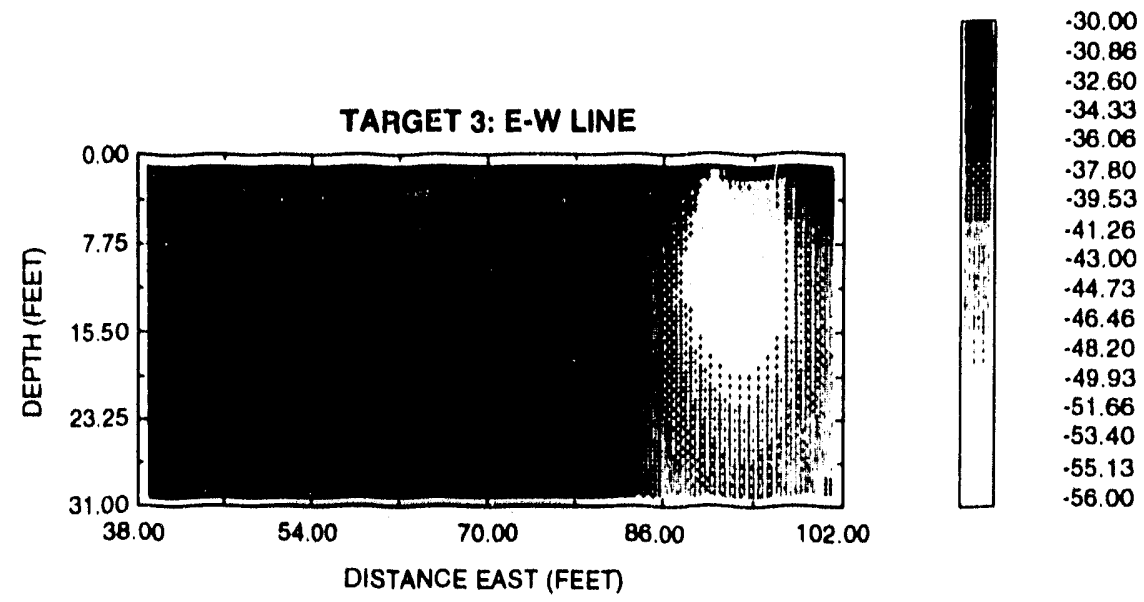

Figure 29: Spatial distribution of the log likelihood function, displayed as gray levels, below magnetometer line E3.

ORNL.DWG $93-6255$
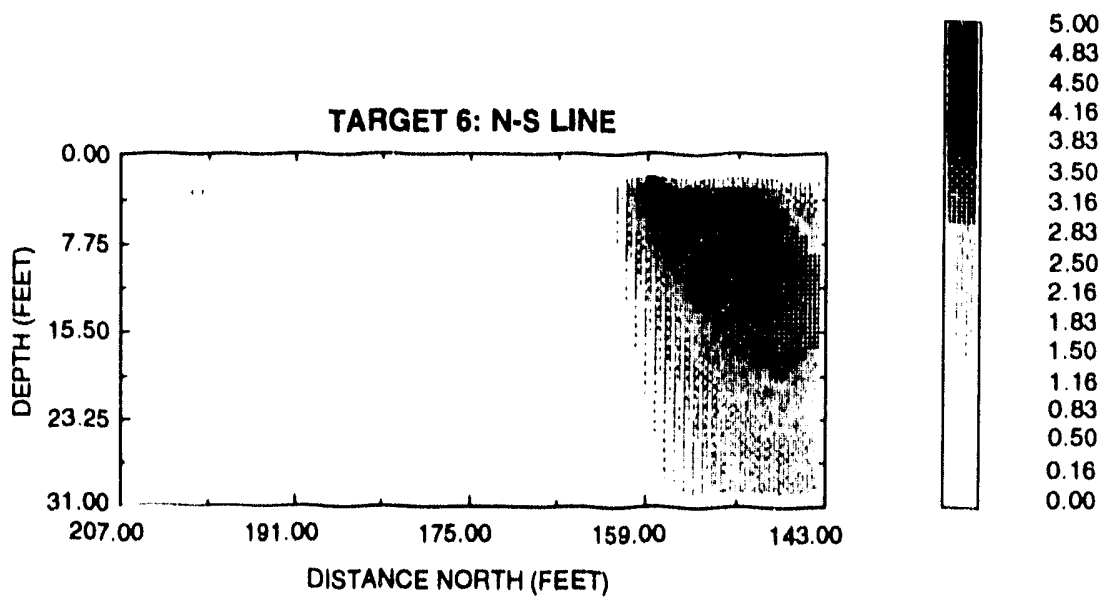

Figure 30: Spatial distribution of the log likelihood function, displayed as gray levels, below magnetometer line $\mathrm{N} 6$. 

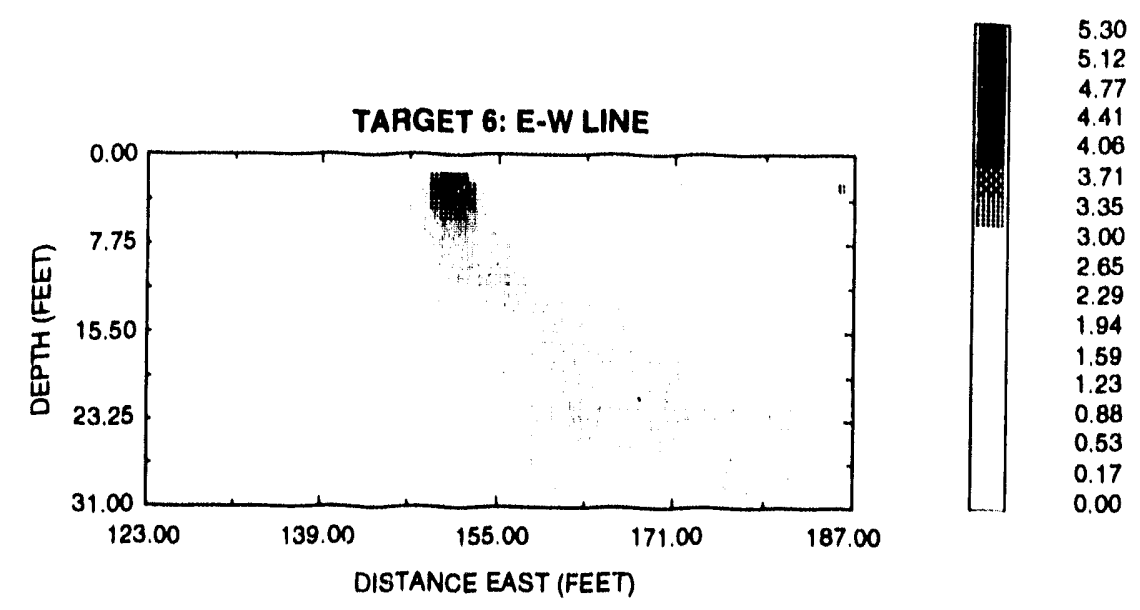

Figure 31: Spatial distribution of the log likelihood function, displayed as gray levels, below magnetometer line $\mathrm{E} 6$.
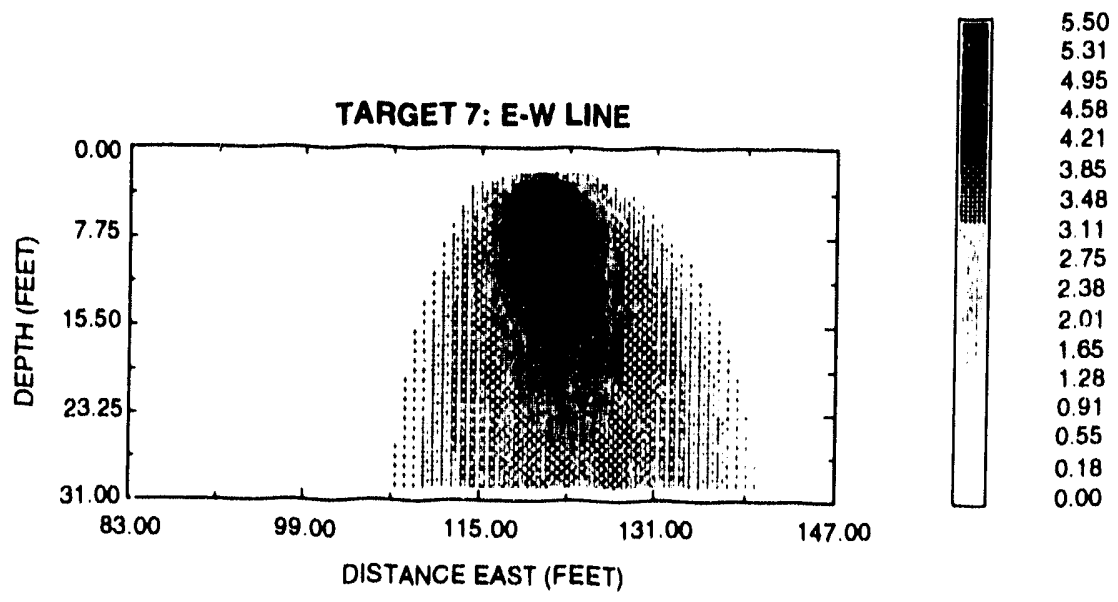

Figure 32: Spatial distribution of the log likelihood function, displayed as gray levels, below magnetometer line $\mathrm{E}$. 
ORNI DWG 93.6040
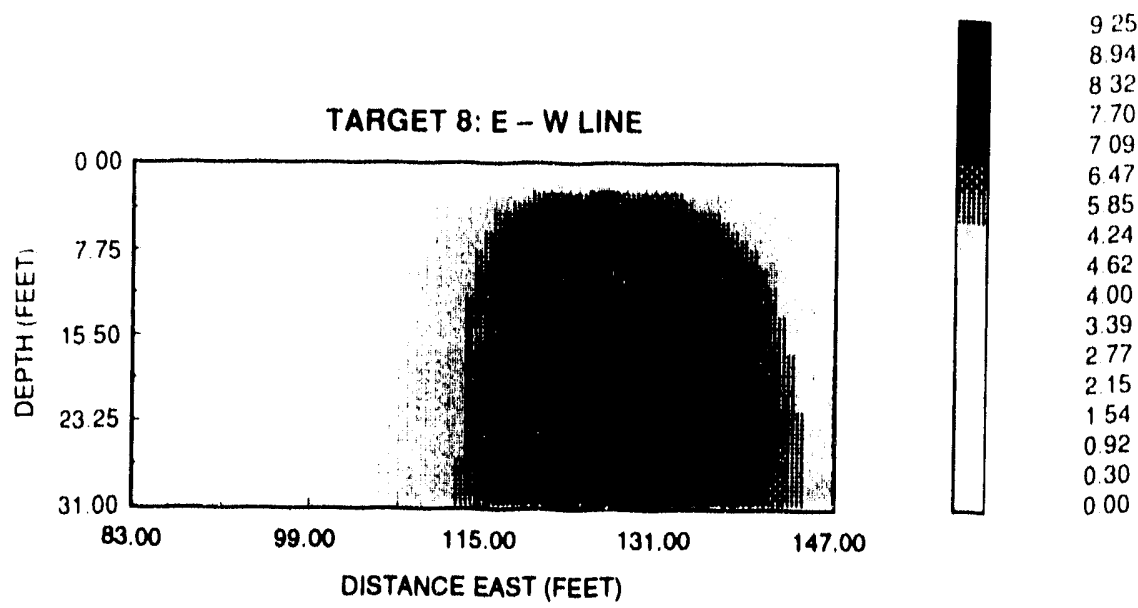

Figure 33: Spatial distribution of the log likelihood function, displayed as gray levels, below magnetometer line ES.

ORNL.DWG 93.6254
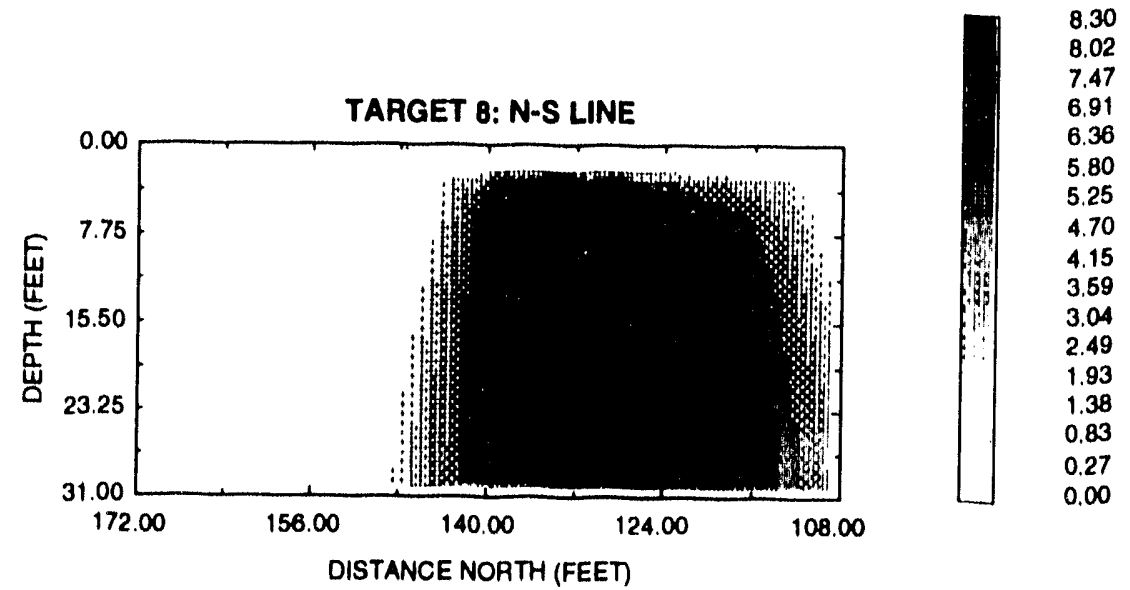

Figure 34: spatial distribution of the log likelihood function, displayed as gray levels, below magnetometer line N8. 


\begin{tabular}{|c|c||c|c|c|c|c|}
\hline \multirow{2}{*}{$\begin{array}{c}\text { Target } \\
\text { Number }\end{array}$} & \multicolumn{2}{c||}{ Operating } & \multicolumn{2}{c|}{ Target Location (ft.) } & \multicolumn{2}{c|}{ Target Dimensions (ft.) } \\
\cline { 3 - 7 } & Frequency (Hz.) & East & North & Depth & East-West & Vertical \\
\hline 6 & 1280 & 150. & 160. & 1.5 & 0.07 & 1.0 \\
6 & 1600 & 150. & 160. & 1.5 & 0.26 & 1.0 \\
7 & 4500 & 120. & 90. & 1.5 & 0.07 & 1.0 \\
7 & 9600 & 120. & 90. & 1.5 & 0.07 & 1.0 \\
\hline
\end{tabular}

Table 3: Summary of the results of the application of a maximum likelihood estimator: to EMI data.

1 boom orientation was fixed. The simulation of EMI data should properly be modeled by a vector equation rather than the scalar equation $[\mathrm{Eq}$. (17)] used in Sect. 2.3. The consideration of a vector governing equation would allow the explicit representation of the orientation of the coil axes in the tool (boom orientation), an aspect that can not be accommodated within the scalar theory. The scalar model used was likely a better representation for east-west transects than for north-south transects.

Table 3 is a summary of the finding from application of the maximum likelihood estimator to the EMI data. A comparison of the magnetometer and EMI results (Tables 2 and 3) reveals only slight differences between these two tools. For both targets, the EMI results estimate target depths slightly, but consistently, shallower than the magnetometer results. For Target 6 , both EMI frequencies yielded the same estimated vertical extent of the target which, in turn, matched that estimated from the magnetometer data. The estimated horizontal dimension of the Target 6 was smaller for the EMI frequencies than the magnetometer with higher frequency estimate more closely matching the magnetometer result. For Target 7, there were differences between the two EMI frequencies, with EMI estimate of vertical target extent being smaller than that for the magnetometer. Horizontal target dimensions estimated by EMI and magnetometry can not be directly compared since the magnetometer result is based on a north-south line and the EMI result is based on an east-west line.

Figures 35 and 36 are gray scale images of spatial variations in $F_{L}$ below an east-west line over the top of Target 6 for the 1280 and $1600 \mathrm{~Hz}$. data, respectively. These two images are quite similar with both showing a strong anomaly at the assumed location for Target 6 (the black area in the upper right) as well as a very weak feature in the upper left. The image at $1600 \mathrm{~Hz}$. is, as expected, somewhat sharper than that at 1280 Hz. Figure 37 is a similar gray scale image for the $4800 \mathrm{~Hz}$. analysis at Target 7 . A comparison of the images of log likelihood function between the magnetometer (Figs. 31 and 32) and EMI (Figs. 35-37) results indicates that EMI offers better spatial resolution than magnetometry particularly in the horizontal direction. 


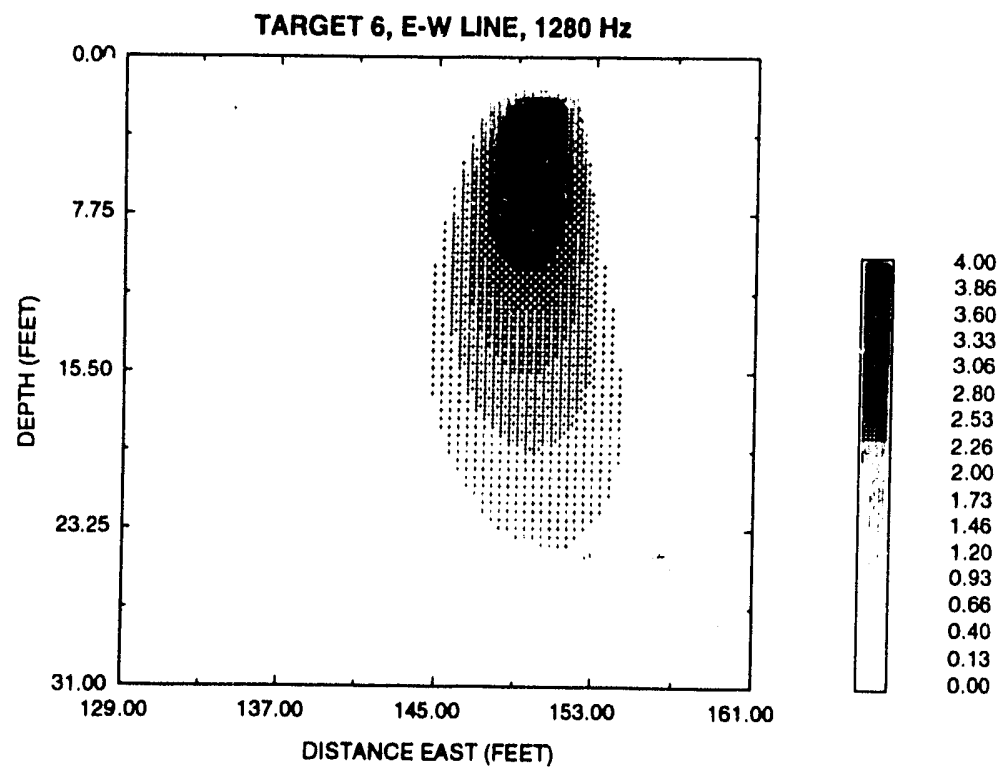

Figure 35: Spatial distribution of the log likelihood function, displayed as gray levels, below Target 6 fro. $11280 \mathrm{~Hz}$. EMI data.

ORNL-DWG $93-6248$
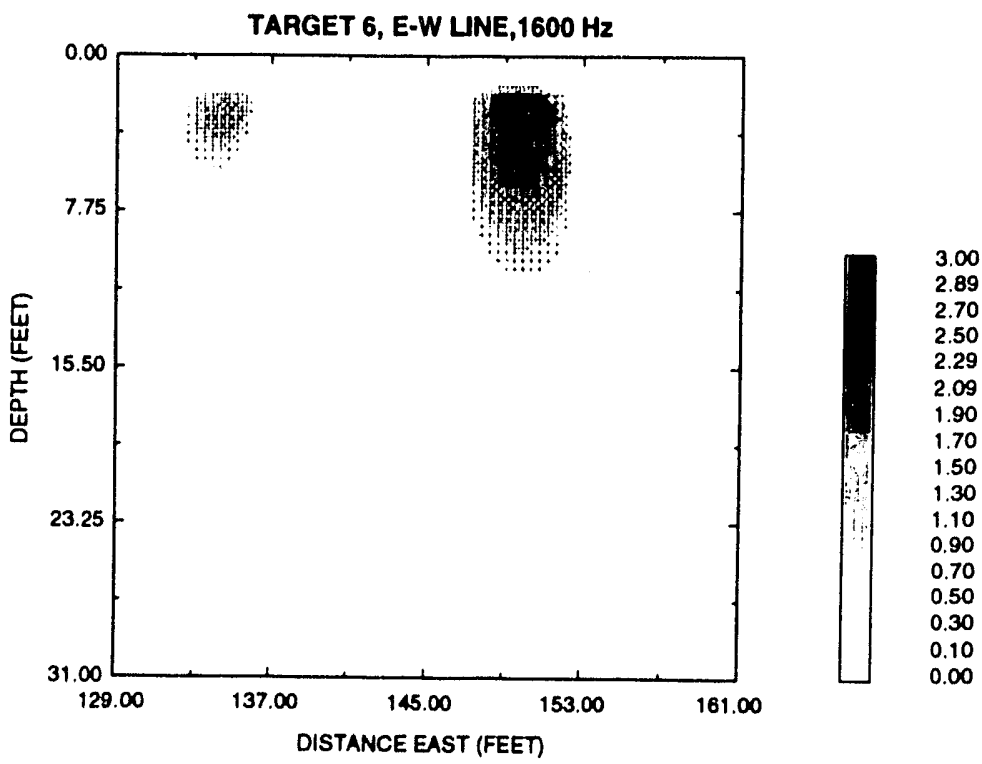

Figure 36: Spatial distribution of the log likelihood function, displayed as gray levels, below Target 6 from $1600 \mathrm{~Hz}$. EMI data. 
ORNL.DWG $93-6249$
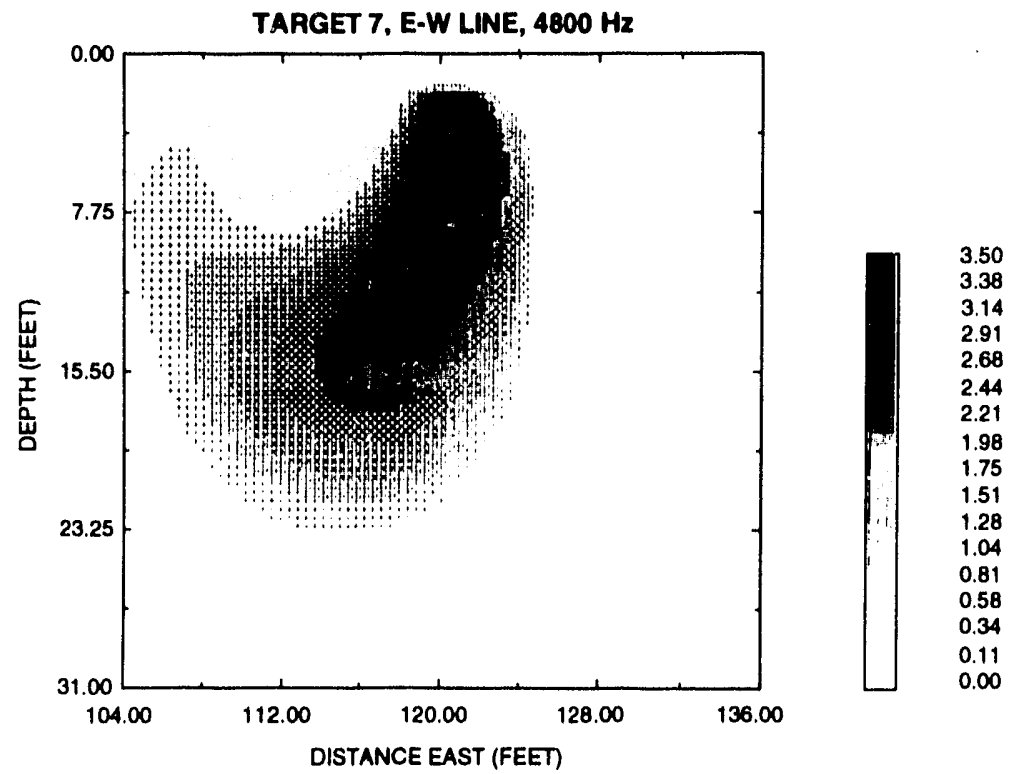

Figure 37: Spatial distribution of the $\log$ likelihood function, displayed as gray levels, below Target 7 from $4800 \mathrm{~Hz}$. EMI data. 


\section{Conclusions and Recommendations}

Buried target detection algorithms have been developed for estimating the size and location of buried features using EMI and magnetometry data. These algorithms were applied to data acquired at a test site containing inert buried ordnance. The field study, as planned, was to focus on EMI data with magnetometry being a secondary technique. It was further planned that a gradient flux gate magnetometer would be the main instrument for magnetic surveys. During the course of the survey, it was discovered that the two EMI tools tested, the GEM1 and the EM 31, proved relatively insensitive to the buried targets of interest. A similar discovery was made with respect to the gradient magnetometer. As a result, the most useful tool in this study proved to be a proton precession magnetometer.

It appears that all buried ordnance were identified with the proton precession magnetometer and that the application of the maximum likelihood estimator algorithm provided reasonable estimates of target location and depth. The estimates of target size are probably not as accurate; however, these are likely within a factor of two of the correct value. The errors in target dimension estimation are probably systematic in the sense that relative returned size estimates of various targets are reasonably accurate when comparing one target to another, (eg., target 10 is three times as long as target 26) even if the absolute sizes are in error. Only selected targets could be located with the EMI tool; however, it is most important to note that the maximum likelihood estimator could locate and size these targets with at least the same, if not better, accuracy as the magnetometer. Furthermore, these EMI targets were located despite the fact that EMI anomalies could not be discerned by conventional interpretation. It is believed that the limitations in size estimation for both EMI and magnetometry are a result of modeling the targets as two-dimensional projections within a vertical cross-section rather than properly treating them as three-dimensional. This was a necessary simplification at this early stage of technique development.

Based on the work to date, it is believed that the use of the computationally efficient maximum likelihood estimator to process EMI and magnetometer data is a viable method for the enhanced characterization of sites known or believed to contain buried ordnance. While EMI did not perform as well as magnetometry, it should not be eliminated from future consideration. The test range at Indian Head was a magnetically clean site that did not have any naturally occurring spatial variations in magnetic permeability. As result, magnetometry alone could be used with confidence that all anomalies are manmade features. This will not always be the case, particularly in the west and south-west where there are sites with numerous magnetic rocks. These naturally occurring features will not produce an EMI response and, for this reason, EMI is expected to be important in, at least, the minimization of false positive finding. Furthermore, EMI should, in principle, provide better spatial resolution. It is recommended that further work in EMI be pursued. Initially, this step in this process would be to collect data over several known targets at many combinations of frequency and spatial sampling. It may, in 
fact, be found that existing EMI tools are adequate for the subject application provided that data acquisition is optimized. A similar optimization study would be useful for magnetometry. Such a study was planned for the beginning of the Indian Head field investigation; unfortunately, no one was available who could identify the locations of known targets.

Further efforts in algorithm development are also important. As a result of the uncertainty in the feasibility of EMI, this should focus on magnetometry. Specific recommendations are to extend and test a three-dimensional target algorithm and to develop computationally efficient procedures to process data that has been acquired at irregular spatial intervals. The later concern is important because many sites are not maintained in the manner Indian Head is and the presence of vegetation may prevent the acquisition of data over a uniform sampling grid. Another potential path for algorithm development is the use of measurements as data templates. Recalling the data template development given in Sect. 2, the GPST provided an analytic relationship between the target located at an arbitrary position and the same target at a reference location. This same shift rule can be applied to measured data provided that the size, location, and orientation of the target is well known. This would totally eliminate the need to model the data templates and, in doing so, also eliminate any errors introduced by such modeling. 


\section{INTERNAL DISTRIBUTION}

1. J.B. Cannon

2. M.A. Kuliasha

3. R.M. Reed

4. R.B. Shelton

5.-14. A.J. Witten
15. ORNL Patent Office

16. Central Research Office

17. Document Reference Section

18. Labcratory Records (RC)

19.-20. Laboratory Records Dept.

\section{EXTERNAL DISTRIBUTION}

21. Dr. Douglas R. Bohi, Director, Energy and Natural Resources Division, Resources for the Future, 1616 P Street, Washington, DC 20036

22. Dr. Thomas E. Drabek, Professor, Department of Sociology, University of Denver, Denver, Colorado 80208-0209

23. Calvin MacCracken, President, Calmac Manufacturing Corporation, 101 West Sheffield Avenue, P. O. Box 710, Englewood, New Jersey 07631

24.-26. Kelly Rigano, U. S. Army Environmental Center, CETHA-TS-S, Aberdeen Proving Ground, Maryland 21010-5401

27. Jacqueline B. Shrago, Director, Office of Technology Transfer, 405 Kirkland Hall, Vanderbilt University, Nashville, Tennessee 37240

28. George F. Sowers, P.E., Senior Vice President, Law Companirs Group, Inc., 114 Townpark Drive, Suite 250, Kennesaw, Georgia 30144-5529

29.-31. Jerry Snyder, Attention: (Code 50) Naval Ordnance Disposal Technology Center, 2008 Stump Neck Road, Indian Head, Maryland 20640-5070

32. Dr. C. Michael Walton, Paul D. and Betty Robertson Meek Centennial Professor and Chairman, Department of Civil Engineering, College of Engineering, The University of Texas at Austin, Cockrell Hall, Suite 4.2, Austin, Texas 78712

33.-34. OSTI, U. S. Department of Energy, P. O. Box 62, Oak Ridge, Tennessee 37831

35. Office of Assistant Manager for Energy Research and Development, DOE/ORO, P. O. Box 2001, Oak Ridge, Tennessee 37831-8600 
